\title{
Mitochondrial complex II is a source of the reserve respiratory capacity that is regulated by metabolic sensors and promotes cell survival
}

\author{
J Pfleger ${ }^{1}, \mathrm{M} \mathrm{He}^{1}$ and $\mathrm{M}$ Abdellatif*,
}

The survival of a cell depends on its ability to meet its energy requirements. We hypothesized that the mitochondrial reserve respiratory capacity (RRC) of a cell is a critical component of its bioenergetics that can be utilized during an increase in energy demand, thereby, enhancing viability. Our goal was to identify the elements that regulate and contribute to the development of RRC and its involvement in cell survival. The results show that activation of metabolic sensors, including pyruvate dehydrogenase and AMP-dependent kinase, increases cardiac myocyte RRC via a Sirt3-dependent mechanism. Notably, we identified mitochondrial complex II (cll) as a target of these metabolic sensors and the main source of RRC. Moreover, we show that RRC, via cll, correlates with enhanced cell survival after hypoxia. Thus, for the first time, we show that metabolic sensors via Sirt3 maximize the cellular RRC through activating cll, which enhances cell survival after hypoxia.

Cell Death and Disease (2015) 6, e1835; doi:10.1038/cddis.2015.202; published online 30 July 2015

During normal/unstressed conditions, the cell runs on a fraction of its mitochondrial bioenergetics capacity, where the difference between the maximum respiratory capacity and basal respiratory capacity is referred to as the spare or reserve respiratory capacity (RRC). In the case when energy demand exceeds supply (e.g., an increase in workload or neuronal activity), the RRC has the potential to increase supply, thus, avoiding an 'ATP crisis'. In accordance, RRC has been shown to correlate with enhanced cell survival ${ }^{1}$ and, conversely, reduced RRC has been associated with neuronal cell death and disease. ${ }^{2}$ RRC is a well-recognized phenomenon; ${ }^{3-9}$ however, its components or the factors that regulate it remain unknown, or, at best, minimally defined. Not surprisingly, one of the known factors that influence the extent of the RRC is substrate availability. ${ }^{7}$

One potential source of RRC is a regulated increase of substrate entry into the TCA cycle that is synchronized with an increase in the electron transport chain (ETC) activity. Interestingly, mammalian complex II (cll) has the unique characteristic of being a common component that links the TCA cycle and the ETC and its role in cell survival and death is well established. For example, inactivating mutations in the subunit A (SDHA) are associated with Leigh's syndrome, which is a progressive neurodegenerative disease associated with neuronal cell death. ${ }^{10}$ Likewise, at least one case report shows that a mutation in cll is associated with heart failure, ${ }^{11}$ while in Drosophila a mutation in Sdhb causes an increase in ROS production and early mortality. ${ }^{12}$ In contrast, inhibition of cll during ischemia/reperfusion attenuates ROS-induced damage. ${ }^{13}$ Indeed, while inhibiting cll has been shown to induce apoptosis, ${ }^{14}$ it is also recognized as an apoptosis sensor. ${ }^{15}$ One mechanism that has been described for cllinduced apoptosis involves its disassembly in the low $\mathrm{pH}$ environment of distressed cells that results in excessive production of ROS from the Sdha. ${ }^{16,17}$ Thus, these results would suggest that a fully assembled cll is critical for cell health and survival, while the disassembled form participates in cell demise. In this report, we show that holo-cll is the source of the RRC, which increases the cells' resistant to cell death.

\section{Results}

The RRC is dependent on the metabolic substrate in a cell type-dependent manner. Our first aim was to assess mitochondrial bioenergetics in live cells and the influence of metabolic substrates on oxygen consumption rates (OCRs) during normoxia versus post-hypoxia. In Figure 1, we measure the OCR of live neonatal cardiac myocytes maintained in atmospheric $\mathrm{O}_{2}$ levels. The characteristics of these cells include spontaneous contraction in culture, high

\footnotetext{
${ }^{1}$ Department of Cell Biology and Molecular Medicine, Rutgers-New Jersey Medical School, Newark, NJ 07103, USA

*Corresponding author: M Abdellatif, Department of Cell Biology and Molecular Medicine, Rutgers-New Jersey Medical School, 185 S Orange Avenue, MSB/Room I-519, Newark, NJ 07103, USA. Tel: 973972 1254; Fax: 973972 7489; E-mail: abdellma@njms.rutgers.edu

Abbreviations: 3NP, 3-nitropropionate; ACC, acetyl CoA carboxylase; AICAR, 5-aminoimidazole-4-carboxamide-1- $\beta$-D-ribofuranosyl 5'-monophosphate; AMPK, AMPactivated protein kinase; ATP, adenosine triphosphate; BSA, bovine serum albumin; cll, complex II; CC, compound C; CPT1, carnitine palmitoyltransferase 1; DAPI, 4',6diamidino-2-phenylindole; DCA, dichloroacetate; DMEM, Dulbecco's Modified Essential Medium; ECAR, extracellular acidification rate; ETC, electron transport chain; FAD, flavin adenine dinucleotide; FBS, fetal bovine serum; FCCP, carbonyl cyanide 4-(trifluoromethoxy)phenylhydrazone; GAPDH, glyceraldehyde-3-phosphate dehydrogenase; Hif- $1 \alpha$, hypoxia inducible factor 1 alpha; iPSC-CM, induced pluripotent stem cell-derived cardiac myocyte; moi, multiplicity of infection; NAD, nicotinamide adenine dinucleotide; OCR, oxygen consumption rate; OXPHOS, oxidative phosphorylation; PBS, phosphate-buffered saline; PDH, pyruvate dehydrogenase; PDK, pyruvate dehydrogenase kinase; PPAR, peroxisome proliferator activated receptor; ROS, reactive oxygen species; RRC, reserve respiratory capacity; SDH, succinate dehydrogenase; SDHAF, succinate dehydrogenase assembly factor; SEM, standard error of the mean; shRNA, short hairpin ribonucleic acid; SIRT, sirtuin Received 12.5.15; revised 15.6.15; accepted 22.6.15; Edited by C Munoz-Pinedo
} 


\section{Neonatal rat myocytes}
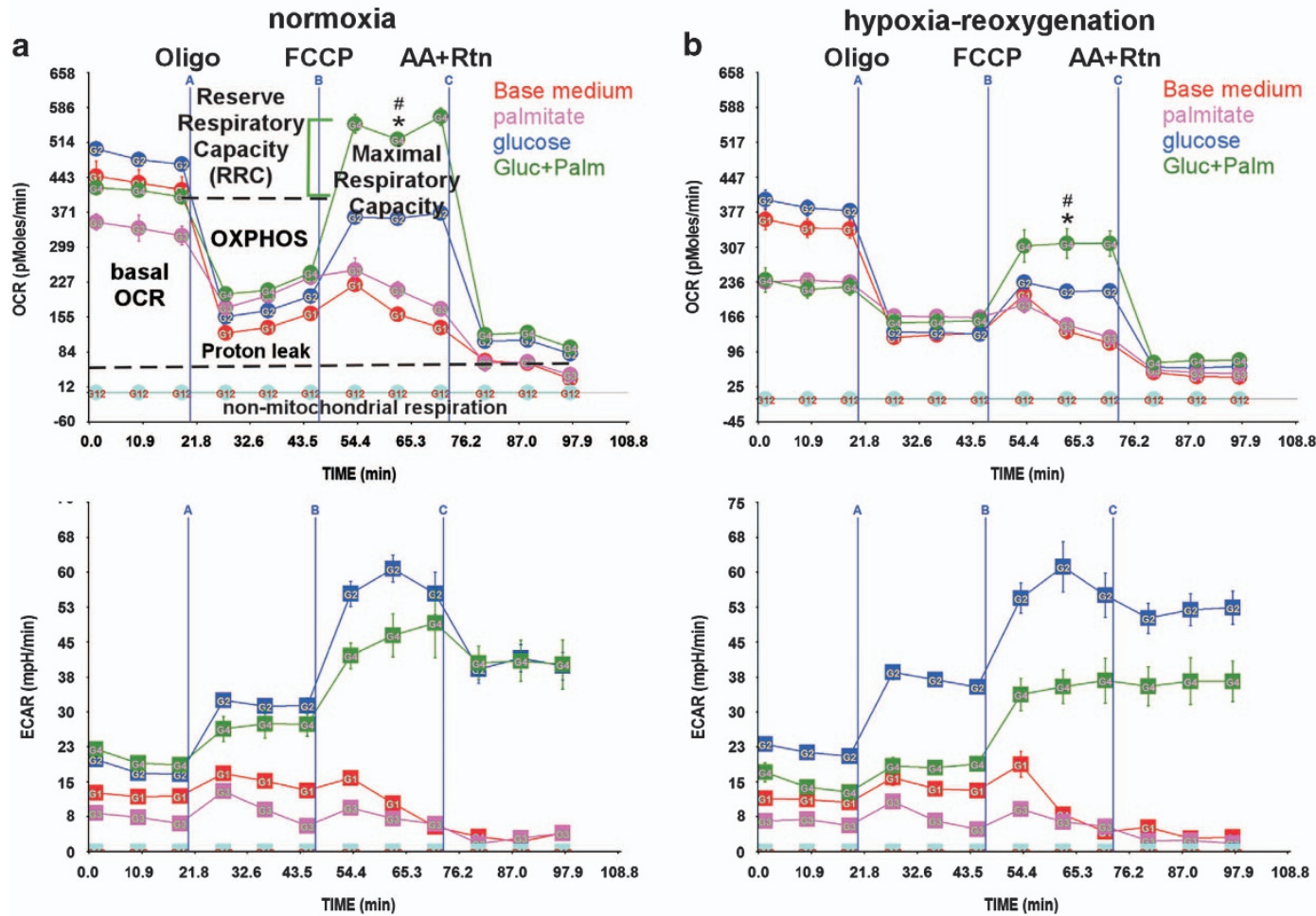

human iPS-derived myocytes
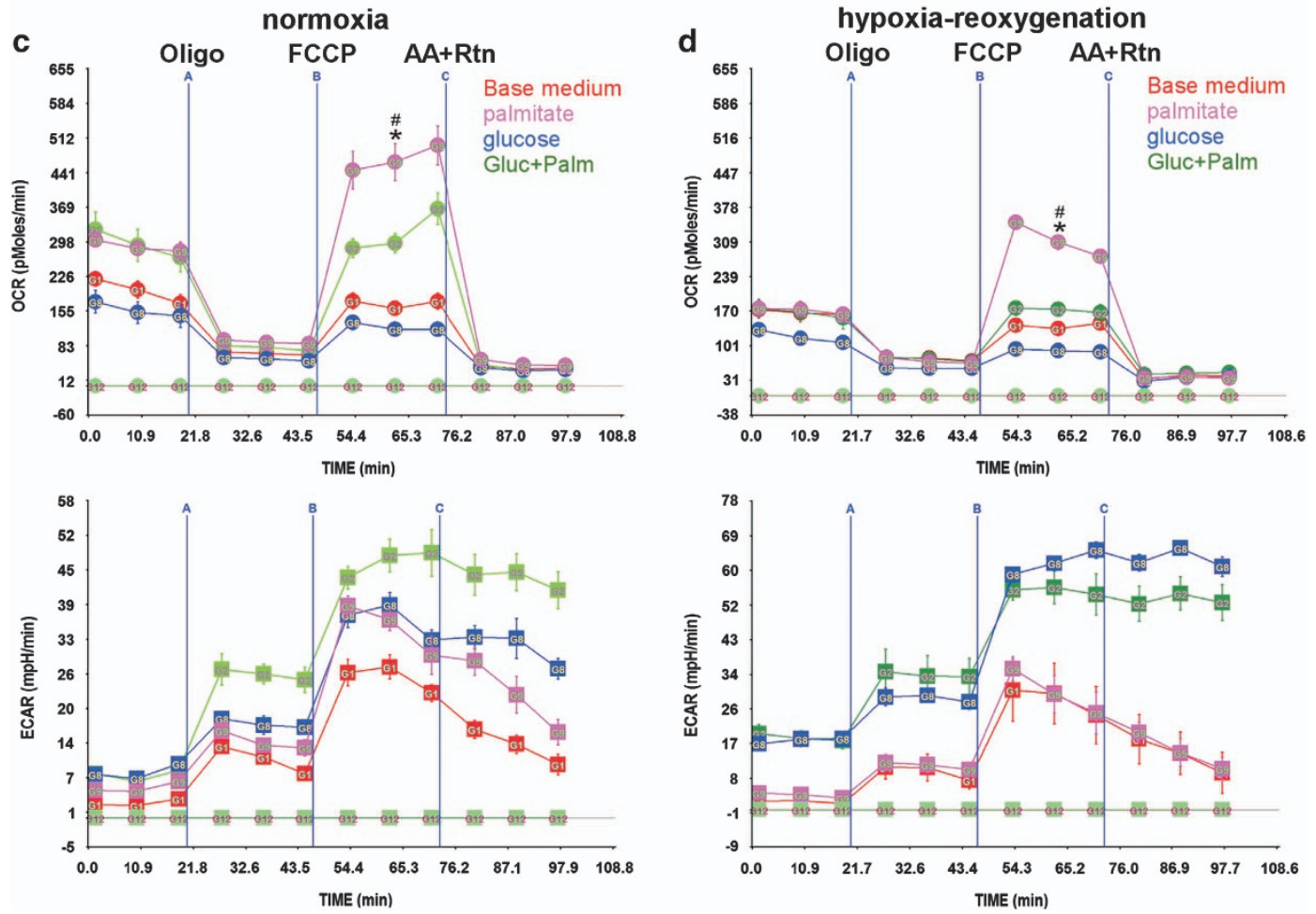
mitochondrial content, and a preference for glucose as a substrate. The data show that basal OCR was the highest (510 pmoles/min/100 000 cells) with glucose in the medium (Figure 1a, upper panel), which was slightly (10-20\%) dampened by palmitate-BSA (Figure 1a, upper panel). On the other hand, the presence of palmitate-BSA or amino acids (base medium with no glucose) alone resulted in 24-33\% lower OCR levels versus control. The injection of oligomycin in the medium revealed the ATP synthesis-linked OCR (OXPHOS), which was proportional to basal OCR.

To determine the maximal respiratory capacity and, thereby, the RRC, the uncoupler p-trifluoromethoxy carbonyl cyanide phenyl hydrazone (FCCP) was injected into the medium at the time point indicated in the graph. The results show that only in the presence of both glucose and palmitate-BSA did the cells have an RRC (1.4- to 2.5-fold over basal OCR). After exposure to hypoxia $\left(<0.1 \% \mathrm{O}_{2}\right)$ for $24 \mathrm{~h}$ (in the presence of complete medium with glucose and fetal bovine serum), the cells were allowed to recover for another $24 \mathrm{~h}$ with the indicated substrates. Notably, under these conditions there was no significant cell death, as determined by the quantitative PCR (qPCR) and live/dead assays (Supplementary Figures S1 and $\mathrm{S} 2)$. As shown, the basal OCR was reduced $(\sim 30 \%)$ with all substrates, accompanied by equivalent decreases in OXPHOS, and the RRC was also downregulated $(60-100 \%)$, with only partial recovery within $24 \mathrm{~h}$ (Figure $1 \mathrm{~b}$, upper panel). Thus, the data demonstrate that basal OCR and $\mathrm{RRC}$ have different substrate requirements, where palmitate is necessary for the development of the latter only, suggesting that these parameters constitute independent entities of the cell's bioenergetics.

To validate the development of RRC in human myocytes, we used human induced-pluripotent stem cell-derived cardiac myocytes (iPSC-CM), which are also spontaneously contracting in culture. Figure 1 shows that basal OCR levels were highest in the presence of palmitate-BSA, whether or not glucose was present ( 300 pmoles $/ \mathrm{min} / 60000$ cells), whereas glucose or amino acid alone resulted in 33-50\% lower OCR levels (Figure 1c, upper panel). In contrast to neonatal myocytes, palmitate-BSA alone produced maximum RRC during normoxia or after hypoxia (1.5- to 2.4-fold over basal OCR), which was diminished by $>80 \%$ by the addition of glucose (Figures 1c and d, upper panels). Also, while monitoring OCR in the cells we are able to simultaneously monitor extracellular acidification rates (ECAR), as an indirect indicator of glycolysis (Figures 1a-d, lower panels). As seen, ECAR levels are highest in the presence of glucose and show a $200 \%$ increase in basal levels and $25 \%$ in maximum capacity after hypoxia, especially in the human myocytes (Figure 1d, lower panel). In short, fatty acids maintain maximum RRC in
hiPSC-CM, which is significantly diminished by glucose, while neonatal myocytes strictly require both fatty acids and glucose to develop a reserve.

\section{The reserve respiratory capacity is regulated by pyruvate} dehydrogenase kinases. A major aspect of metabolism that is modified during hypoxia is the inhibition of glucose oxidation via hypoxia-inducible factor 1-alpha (Hif-1a)mediated increase in Pdk1. Therefore, we examined the expression pattern of the Pdk isoforms during normoxia and after hypoxia/reoxygenation with different metabolic substrates, as well as, immediately following $24 \mathrm{~h}$ of hypoxia in complete medium. As seen in Figure 2, the different substrates did not have any impact on the expression levels of Pdks 1-3 during normoxia, with a small but significant increase in Pdk4 in the presence of palmitate (Figure 2a), while hypoxia increased the levels of Pdk1 (9-fold) and Pdk4 (2.5-fold) (Figure 2b). After $24 \mathrm{~h}$ of reoxygenation, Pdk1 levels returned to basal values, whereas Pdk4 continued to increase ( 10-fold) in the presence of palmitate (Figure $2 \mathrm{c})$. The latter could explain the lack of full metabolic recovery $24 \mathrm{~h}$ after hypoxia/reoxygenation.

We hypothesized that Pdks are negative regulators of the RRC. To test this, we overexpressed Pdk1, Pdk4 or Hif-1a, which induces the expression of Pdk1, in neonatal myocytes, in the presence of palmitate-BSA plus glucose. The results of this experiment show that both Pdk1 and Hif-1a completely eliminated the RRC, with minimal $(\sim 15 \%)$, but insignificant, reduction of basal OCR (Figure $2 \mathrm{~d}$ ), which adjusts via utilization of amino acids (see Figure 1a). Notably, Pdk4 had little effect on RRC under these conditions.

Since RRC requires both glucose and fatty acid, we predicted that inhibition of mitochondrial uptake of fatty acid would similarly abrogate RRC. Figure $2 e$ proves this to be true, via inhibiting carnitine palmitoyltransferase 1 (Cpt1) with etomoxir, which resulted in complete abrogation of RRC and $\sim 20 \%$, but insignificant, reduction in basal OCR. These results confirm that both glucose and fatty acid function synergistically to generate an RRC in neonatal myocytes, and emphasize the fact that basal OCR and RRC have unique metabolic requirements and regulators.

To confirm the role of Pdks in regulating RRC, we treated the cells with the Pdk inhibitor dichloroacetate (DCA), which activates glucose oxidation. ${ }^{18}$ The results show that DCA modestly increased basal OCR (22-25\%) during normoxia in cells that were supplied with glucose plus palmitate, but not glucose only (Figure 2f, upper panel). On the other hand, it more robustly increased RRC by 1.66 - and 1.56 -fold under those same conditions, respectively. Furthermore, hypoxia/ reoxygenation reduced basal OCR and RRC of DCA-treated

\footnotetext{
Figure 1 The RRC is differentially regulated by glucose and fatty acid oxidation in neonatal rat myocytes and human iPSC-C. (a-d) Neonatal rat cardiac myocytes (a and $\mathbf{b})$ or human IPSC-CM (c and $\mathbf{d}$ ) were incubated for $24 \mathrm{~h}$ in complete growth medium under normoxic (atmospheric $\left.\mathrm{O}_{2}\right)(\mathbf{a}$ and $\mathbf{c})$ or hypoxic $\left(<1 \% \mathrm{O}_{2}\right)(\mathbf{b}$ and $\mathbf{d})$ conditions. After this period, the medium was replaced with glucose- and fatty acid-free base medium, or that containing $17.5 \mathrm{mM}$ glucose, $100 \mu \mathrm{M}$ palmitate-BSA or $17.5 \mathrm{mM}$ glucose plus $100 \mu \mathrm{M}$ palmitate-BSA (Gluc+Palm), as indicated, in normoxic conditions, for another $24 \mathrm{~h}$. The medium was then replaced with serum-free XF medium containing the indicated substrates. The mitochondrial stress test was then performed as described in Methods and Materials, $n=4-6$, each experiment performed twice. For (a and $\mathbf{b}$ ), error bars represent S.E.M., ${ }^{\star} P<0.05$ max OCR versus basal OCR for Gluc+Palm at the time point indicated; ${ }^{\#} P<0.05$ max OCR for Gluc+Palm versus max OCR of base medium. For (c and d), error bars represent S.E.M., ${ }^{*} P<0.05$ max OCR versus basal OCR for palmitate at the time point indicated; ${ }^{\#} P<0.05$ max OCR for palmitate versus max OCR of base medium
} 

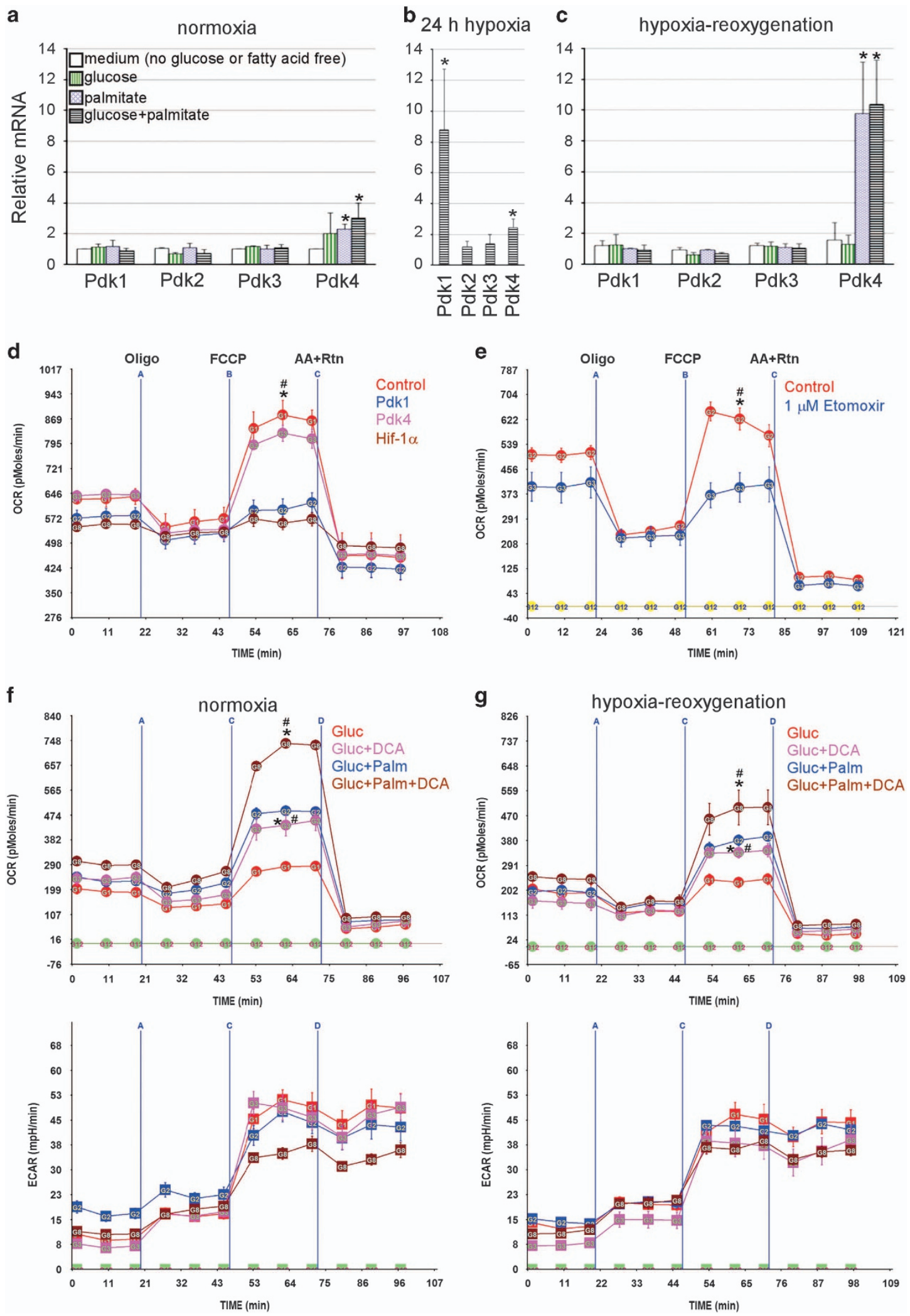
and untreated cells equally, however, only the DCA-treated cells, in the presence of glucose plus palmitate, retained values that are equivalent to untreated cells during normoxia, as seen in Figure $2 \mathrm{~g}$ (upper panel). It should be noted that none of the treatments had a significant effect on ECAR (Figures $2 f$ and g, lower panels). This suggests that the nonlethal hypoxia-induced mitochondrial dysfunction may be attributable to the sustained increase in Pdk activity and is largely preventable via its inhibition.

The reserve respiratory capacity is regulated by AMPK. AMPK is a metabolic sensor ${ }^{19}$ that can acutely enhance fatty acid oxidation via an increase in acetyl-CoA carboxylase 2 phosphorylation-mediated activation of Cpt1. ${ }^{20}$ Thus, we hypothesized that AMPK, through activating Cpt1 may contribute to RRC and/or basal OCR during recovery from hypoxia. To test this, we treated the myocytes with either an adenosine analog activator of AMPK (5-amino-1- $\beta$-D-ribofuranosyl-imidazole-4-carboxamide (AICAR)) or AICAR plus the AMPK inhibitor, compound $C$ (CC). The results show that AICAR increased reserve from 1.34-fold to 1.9-fold, but had no effect on basal OCR during normoxia (Figure 3a, upper panel). The addition of CC inhibitor completely abolished the effect of AICAR on reserve, and like etomoxir, had no effect on basal OCR, thus, confirming that the effects of AICAR are indeed AMPK dependent. A similar effect was seen after hypoxia/reoxygenation, where the RRC was 2.5 -fold versus basal OCR (Figure 3b, upper panel). However, unlike what we have seen with DCA in Figure 2, AICAR did not rescue the reduction in basal OCR. Moreover, AICAR's effect on RRC was only elicited in the presence of glucose plus palmitate, but not palmitate alone (Figure $3 c$, upper panel). This is expected in the neonatal myocytes, as palmitate strictly requires the presence of glucose to enhance $\mathrm{RRC}$.

Since the time point used in the latter experiments for activation AMPK is $48 \mathrm{~h}$, there is a possibility that the effects of AICAR are mediated through a transcriptional, peroxisome proliferator-activated receptor alpha (Ppara)-dependent, mechanism. qPCR results confirm that AICAR enhanced the expression of Ppara, peroxisome proliferator-activated receptor gamma, coactivator 1 alpha (Pgc-1a), and Cpt1 during normoxia (Figure 3e). The same set of genes was also induced by hypoxia, however, $24 \mathrm{~h}$ post hypoxia, while Pgc$1 a$ and Cpt1 remained higher than control, Ppara was significantly lower, and recovered by treatment with AICAR (Figure 3e). A pattern similar to Ppara expression was seen with transcription factor A, mitochondrial (Tfam) and mitochondrial numbers (Figure $3 f$ ). Therefore, to determine the role of Ppara in mediating the effect of AICAR on RRC, we measured OCR in the presence of short hairpin RNA (sh) targeting Ppara (Ppara-sh). Treatment with this construct resulted in complete inhibition of AICAR-induced upregulation of Ppara ( 0.5-fold of basal), and a 3.5-fold increase in Pgc-1a mRNA (mechanism unknown), but had no effect on Cpt1 or Tfam (Figure $3 \mathrm{~g}$ ). This was accompanied by $70 \%$ inhibition of AICAR-induced RRC (Figure 3d, upper panel). The results show that after hypoxia, fatty acid oxidation may be compromised due to a reduction in Ppara, and that this could be prevented by AMPK activation, resulting in restoration of RRC.

\section{Regulation of the reserve respiratory capacity in human} iPSC-derived cardiac myocytes. The hiPSC-CM are $>95 \%$ differentiated myocytes that are spontaneously beating in culture (Figure 4a). As shown in Figure 1, these cells have an RRC that is dependent on fatty acid only and is dampened by glucose. So while the neonatal myocytes benefit from the addition of DCA as it enhances both basal OCR and RRC, it was unclear how the hiPSC-CM would benefit from an increase in glucose oxidation. Interestingly, our results show that DCA increases RRC before ( 2.5-fold, Figure $4 \mathrm{~b}$, upper panel) and after hypoxia ( 1.7-fold, Figure 4c, upper panel). The treatment had little impact on basal OCR during normoxia, but restored the decrease seen after hypoxia. In contrast, AICAR did not have a significant effect on the OCR in these cells, plausibly due to inherent optimal fatty acid oxidation. On the other hand, AICAR enhanced ECAR, indicating an increase in glycolysis (Figures $4 \mathrm{~b}$ and $\mathrm{c}$, lower panels). These results indicate that activation of pyruvate dehydrogenase in either neonatal cardiac myocytes or hiPSC-CM has the capacity to significantly increase RRC and, thereby, restore the cells' bioenergetics after exposure to hypoxia.

Figure 2 The RRC is regulated by pyruvate dehydrogenase kinases. $(\mathrm{a}-\mathrm{c})$ Neonatal rat cardiac myocytes were incubated for $24 \mathrm{~h}$ in complete growth medium under normoxic (atmospheric $\left.\mathrm{O}_{2}\right)(\mathbf{a})$ or hypoxic $\left(<1 \% \mathrm{O}_{2}\right)(\mathbf{b}$ and $\mathbf{c})$ conditions. After this period, RNA was either immediately extracted and subjected to qPCR for the indicated genes (b), or the medium was replaced with one that is glucose and fatty acid free, or one containing $17.5 \mathrm{mM}$ glucose, $100 \mu \mathrm{M}$ palmitate-BSA, or glucose+palmitate-BSA, as indicated, in normoxic conditions, for another $24 \mathrm{~h}$ (a and $\mathbf{c})$. The results were averaged plotted as relative values to those from cells incubated in base medium in normoxic conditions adjusted to 1 (a and $\mathbf{c}$ ) or control normoxia adjusted to 1 (b), $n=3$. Error bars represent standard error of the mean (S.E.M.), ${ }^{*} P<0.05$ versus control. (d) Neonatal cardiac myocyte was incubated for $24 \mathrm{~h}$ in complete growth medium and then infected with a control or adenoviruses (Ad) harboring PDK1, PDK4 or Hif- $1 \alpha$, for $24 \mathrm{~h}$. The medium was then replaced with serum-free XF medium containing $17.5 \mathrm{mM}$ glucose plus $100 \mu \mathrm{M}$ palmitate for $1 \mathrm{~h}$. The mitochondrial stress test was then performed as described in Materials and Methods, $n=4-6$, each experiment was performed twice. Error bars represent S.E.M., ${ }^{*} P<0.05$ max OCR versus basal OCR for control at the time point indicated; ${ }^{\#} P<0.05$ max OCR for control versus max OCR of Pdk1 or Hif- $1 \alpha$ treated. (e) Neonatal cardiac myocytes were cultured for $24 \mathrm{~h}$ in complete growth medium containing vehicle or $1 \mu \mathrm{M}$ etomoxir, a Cpt1 inhibitor. The medium was then replaced with serum-free XF medium containing $17.5 \mathrm{mM}$ glucose plus $100 \mu \mathrm{M}$ palmitate containing vehicle or $1 \mu \mathrm{M}$ etomoxir, for $1 \mathrm{~h}$. The mitochondrial stress test was then performed as described in Materials and Methods, $n=4-6$, each experiment was performed twice. Error bars represent S.E.M., ${ }^{*} P<0.05$ max OCR versus basal OCR for control at the time point indicated; ${ }^{\#} P<0.05$ max OCR for control versus max OCR for etomoxir treated, at the time point indicated (f and $\mathbf{g}$ ). Neonatal rat cardiac myocytes were cultured in complete growth medium containing vehicle or $1 \mathrm{mM}$ DCA and either remained in normoxic conditions $\left(\right.$ atmospheric $\left.\mathrm{O}_{2}\right)(\mathbf{f})$ or were exposed to hypoxia $\left(<1 \% \mathrm{O}_{2}\right)(\mathrm{g})$, for $24 \mathrm{~h}$. At the end of this period, the medium was changed to base medium containing $17.5 \mathrm{mM}$ glucose or $17.5 \mathrm{mM}$ glucose plus $100 \mu \mathrm{M}$ palmitate-BSA containing vehicle or DCA, as indicated, for an additional $24 \mathrm{~h}$. The medium was then replaced with serum-free XF medium containing $17.5 \mathrm{mM}$ glucose or $17.5 \mathrm{mM}$ glucose plus $100 \mu \mathrm{M}$ palmitate-BSA containing vehicle or $1 \mathrm{mM} \mathrm{DCA}$, for $1 \mathrm{~h}$. The mitochondrial stress test was then performed as described in Materials and Methods, $n=4-6$, each experiment was performed three times. Error bars represent S.E.M., ${ }^{*} P<0.05$ max OCR versus basal OCR for DCA-treated cells, at the time point indicated; ${ }^{\#} P<0.05$ max OCR for DCA-treated versus max OCR for untreated, at the time point indicated 
a
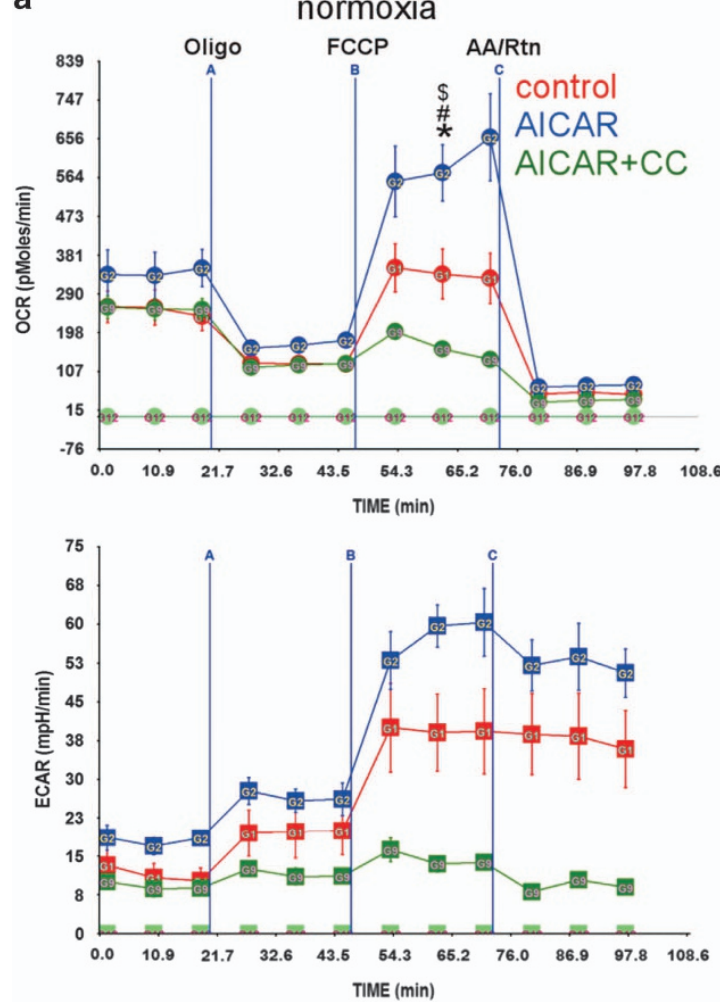

C
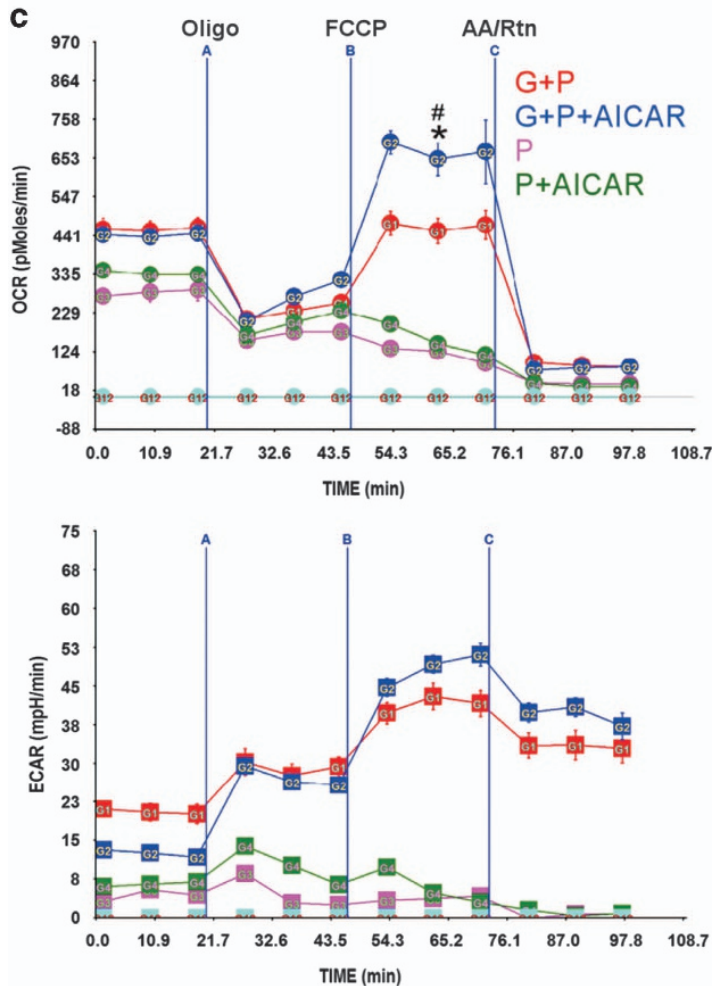

Figure 3 Continued
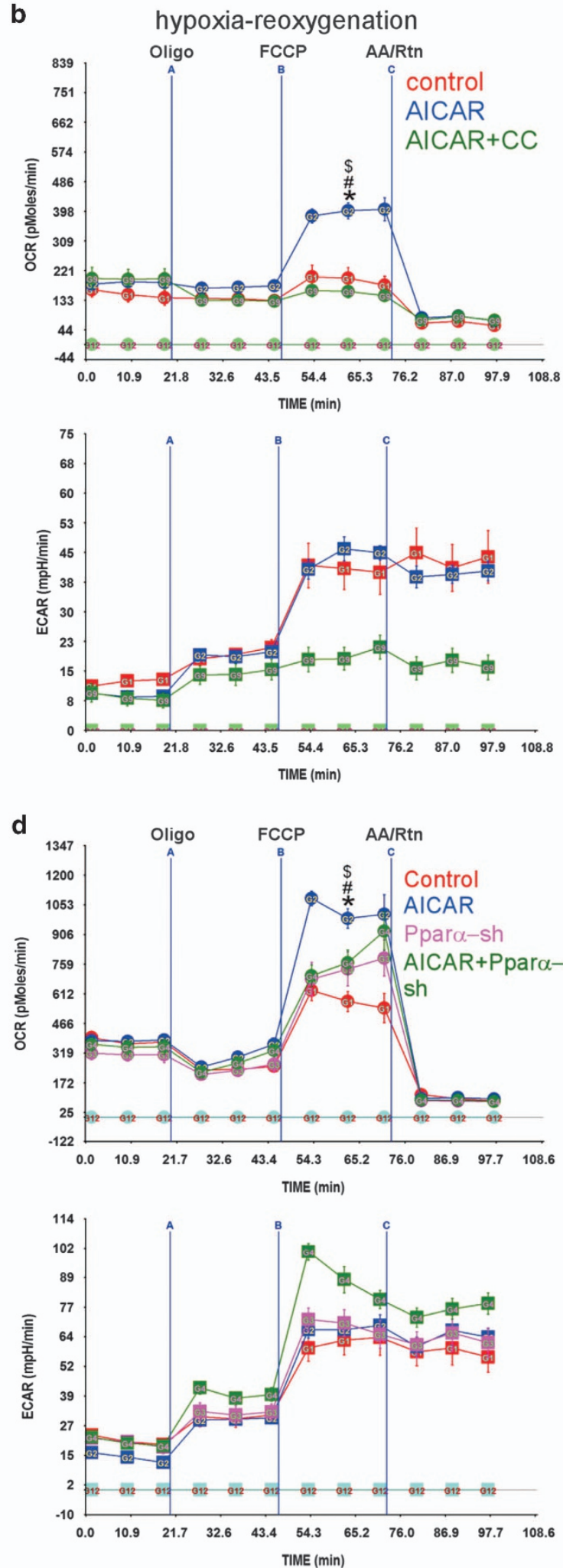

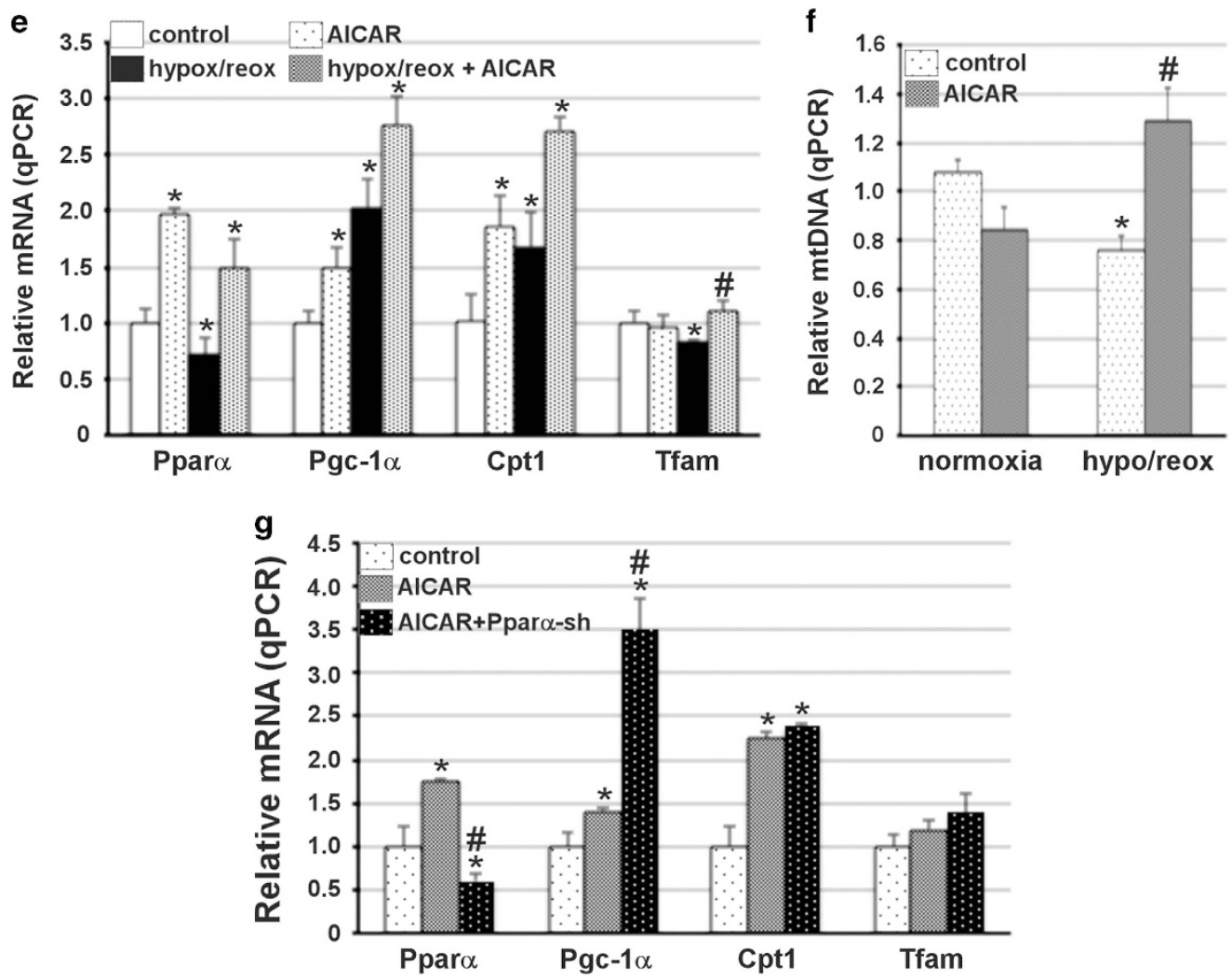

Figure 3 The RRC is regulated by AMPK via Ppar $\alpha$. (a and $\mathbf{b}$ ) Neonatal rat cardiac myocytes were cultured in complete growth medium containing vehicle, $500 \mu \mathrm{M} \mathrm{AICAR}$ or $500 \mu \mathrm{M}$ AICAR plus $5 \mu \mathrm{M}$ compound $\mathrm{C}(\mathrm{CC})$ and either remained in normoxic conditions (atmospheric $\mathrm{O}_{2}$ ) (a) or were exposed to hypoxia $\left(<1 \% \mathrm{O}_{2}\right)$, for $24 \mathrm{~h}$ (b). The medium was replaced with base medium containing $17.5 \mathrm{mM}$ glucose plus $100 \mu \mathrm{M}$ palmitate-BSA containing vehicle, $500 \mu \mathrm{M}$ AICAR or $500 \mu \mathrm{M}$ AICAR plus $5 \mu \mathrm{M}$ CC, for $24 \mathrm{~h}$. The medium was then replaced with serum-free XF medium containing $17.5 \mathrm{mM}$ glucose plus $100 \mu \mathrm{M}$ palmitate-BSA containing vehicle, $500 \mu \mathrm{M}$ AICAR or $500 \mu \mathrm{M}$ AICAR plus $5 \mu \mathrm{M}$ $\mathrm{CC}$, for $1 \mathrm{~h}$. The mitochondrial stress test was then performed as described in Materials and Methods, $n=4-6$, each experiment was performed three times. Error bars represent S.E.M., ${ }^{*} P<0.05$ max OCR versus basal OCR for AICAR-treated cells, at the time point indicated: ${ }^{*} P<0.05$ max OCR for AICAR-treated versus max OCR for control, at the time point indicated; ${ }^{\$} P<0.05$ max OCR for AICAR treated versus max OCR for CC treated. (c) Neonatal rat cardiac myocytes were treated with vehicle or $500 \mu \mathrm{M}$ AICAR in the presence of $17.5 \mathrm{mM}$ glucose plus $100 \mu \mathrm{M}$ palmitate-BSA or $100 \mu \mathrm{M}$ palmitate-BSA, for $24 \mathrm{~h}$. The medium was then replaced with serum-free XF medium containing $17.5 \mathrm{mM}$ glucose plus $100 \mu \mathrm{M}$ palmitate-BSA or $100 \mu \mathrm{M}$ palmitate-BSA containing vehicle or $500 \mu \mathrm{M}$ AICAR, for $1 \mathrm{~h}$. The mitochondrial stress test was then performed as described in Materials and Methods, $n=4-6$, each experiment was performed three times. Error bars represent S.E.M., ${ }^{\star} P<0.05$ max OCR versus basal OCR for AICAR-treated cells in glucose+palmitate $(\mathrm{G}+\mathrm{P})$, at the time point indicated; ${ }^{\#} P<0.05$ max OCR for AICAR treated versus max OCR for untreated in $\mathrm{G}+\mathrm{P}$, at the time point indicated. (d) Neonatal rat cardiac myocytes were cultured in complete growth medium treated with vehicle or $500 \mu \mathrm{M}$ AICAR in the presence of adenoviral-control shRNA or -shRNA-Ppar $\alpha$ - constructs, for $24 \mathrm{~h}$. The medium was then replaced with serum-free XF medium containing $17.5 \mathrm{mM}$ glucose plus $100 \mu \mathrm{M}$ palmitate-BSA containing vehicle or $500 \mu \mathrm{M}$ AICAR, for $1 \mathrm{~h}$. The mitochondrial stress test was then performed as described in Materials and Methods, $n=4-6$, each experiment was performed two times. Error bars represent S.E.M., ${ }^{*} P<0.05$ max OCR versus basal OCR for AICAR-treated cells, at the time point indicated; ${ }^{\#} P<0.05$ max OCR for AICAR treated versus max OCR for control; $\$ P<0.05$ max OCR for AICAR treated versus max OCR of AICAR- plus shRNA-Ppar $\alpha$-treated, at the time point indicated. (e and $\mathbf{f})$ Neonatal cardiac myocytes were treated as described in (a and $\mathbf{b})$, with AICAR in normoxia versus hypoxia/reoxygenation (hypox/reox), as indicated. Total mRNA (e) and DNA (f) were extracted and subjected to qPCR for the indicated genes $(n=4)$. The results were averaged and plotted as relative values to control medium (no glucose or fatty acids). Error bars represent S.E.M., ${ }^{*} P<0.05$ versus control, ${ }^{\sharp} P<0.05$ versus control hypox/reoxx. (g) Neonatal myocyes were treated with adenoviral shRNA-control and shRNA-Ppar $\alpha$, with or without $500 \mu \mathrm{M}$ AICAR for $24 \mathrm{~h}$ in the presence of $17.5 \mathrm{mM}$ glucose $+100 \mu \mathrm{M}$ palmitate-BSA. Total RNA was extracted and analyzed by qPCR for the indicated genes. The results were averaged and plotted as values relative to control. Error bars represent S.E.M., ${ }^{*} P<0.05$ versus control

Complex II is a source of RRC. In an attempt to identify the source of RRC in the cell, we measured the activities of the respiratory complexes in an electron flow assay. The results reveal that AICAR induced an overall increase in electron flow activity in the uncoupled state (1.5- to 1.7-fold), and, in specific, an increase in cll activity (1.6- to 2-fold). The cllspecific inhibitor 3-nitropropionate (3NP) completely abolished the effect of AICAR on basal flow (cl-cIV), restoring it to baseline values, while completely suppressing cll activity (Figure 5a). A similar effect was observed with DCA (Figure 5b). Notably, complex IV activity varied according to changes in electron flow. It should also be noted that there were quantitative but not qualitative differences in basal and activated cll OCR values between the primary myocyte cultures, as observed in Figures $5 \mathrm{a}$ and b. Consequently, we predicted that the RRC might be a product of an increase in complex II activity. To test this, we measured the effect of AICAR on RRC in the presence or absence of 3NP. As demonstrated in Figure 5c, 3NP completely abolished RRC, in addition to, minimal (15-20\%), but insignificant, effect on basal OCR. Thus, we concluded that an increase in cll activity contributes to formation of RRC via increasing electron flow directly from $\mathrm{FADH}_{2}$-succinate dehydrogenase. 

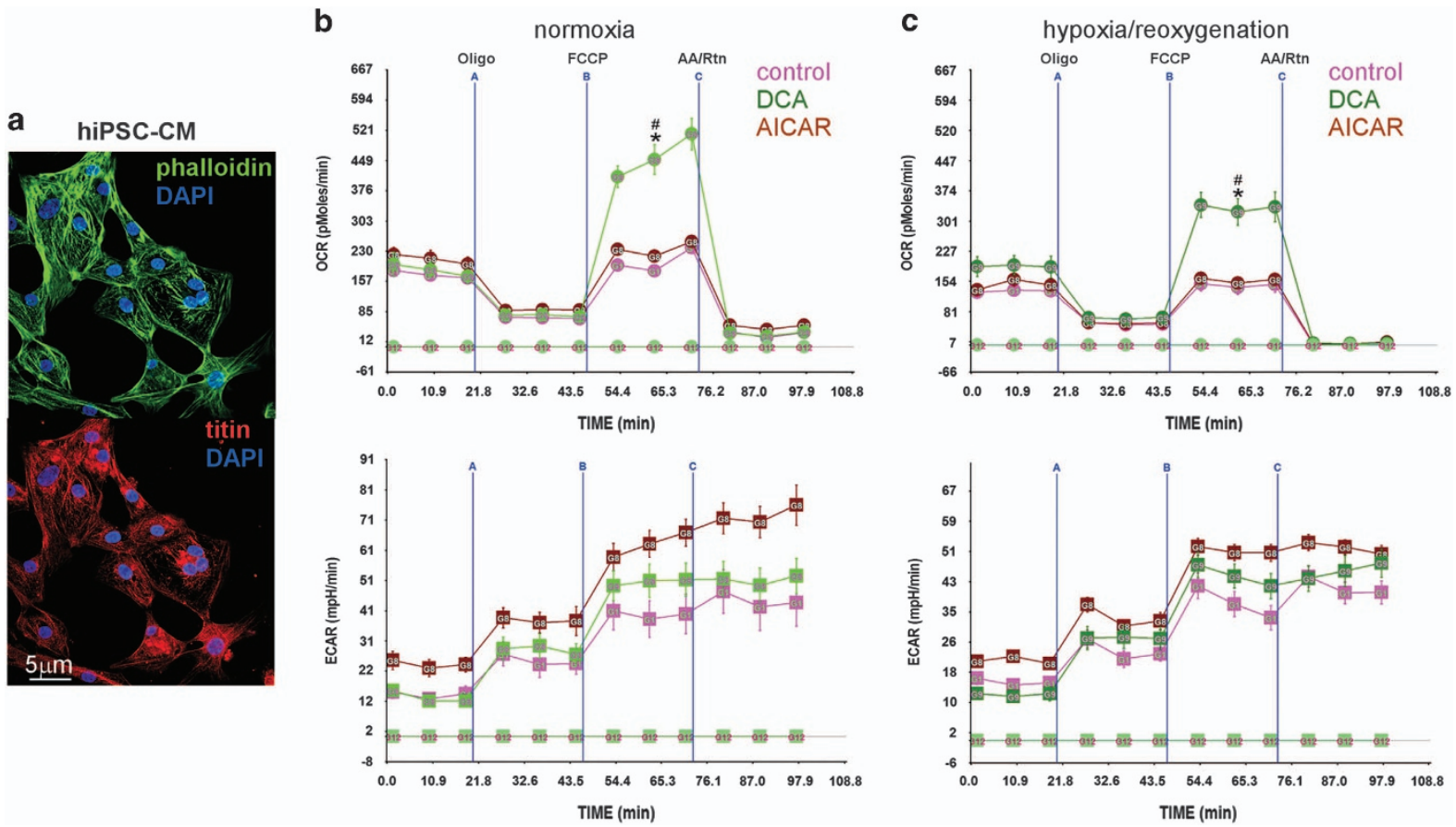

Figure 4 The RRC in human iPSC-CM is regulated by DCA but not by AICAR. (a) Human IPSC-CM were cultured, fixed, permeabilized and incubated with the indicated primary antibodies. They were then incubated with fluorescently labeled phalliodin (upper panel) or secondary antibody (lower panel), mounted with DAPI and imaged by confocal microscopy. (b and c) Human iPSC-CM were cultured in complete growth medium containing vehicle, $1 \mathrm{mM} \mathrm{DCA}$ or $500 \mu \mathrm{M}$ AICAR and either remained in normoxic conditions (atmospheric $\left.\mathrm{O}_{2}\right)(\mathbf{b})$ or were exposed to hypoxia $\left(<1 \% \mathrm{O}_{2}\right)(\mathbf{c})$, for $24 \mathrm{~h}$. At the end of this period, the medium was changed to base medium containing $17.5 \mathrm{mM}$ glucose plus $100 \mu \mathrm{M}$ palmitate-BSA containing vehicle, $1 \mathrm{mM}$ DCA or $500 \mu \mathrm{M}$ AICAR, as indicated, for an additional $24 \mathrm{~h}$. The medium was then replaced with serum-free XF medium containing $17.5 \mathrm{mM}$ glucose plus $100 \mu \mathrm{M}$ palmitate-BSA containing vehicle, $1 \mathrm{mM} \mathrm{DCA}$ or $500 \mu \mathrm{M}$ AICAR, for $1 \mathrm{~h}$. The mitochondrial stress test was then performed as described in Materials and Methods, $n=4-6$, each experiment was performed twice. Error bars represent S.E.M., ${ }^{*} P<0.05$ max OCR versus basal OCR for DCA-treated cells, at the time point indicated; ${ }^{\#} P<0.05$ max OCR for DCA-treated versus max OCR for control, at the time point indicated

We speculated that after a short period of nutrient and oxygen deprivation and before cell demise (as assessed by a live/dead cell assay, Supplementary Figure S2), myocytes will attempt to recover their energy supply by increasing energy production. In accordance, AMPK, through activation of cll, will increase basal OCR and oxidative phosphorylation. This was confirmed after incubating the cells for $1 \mathrm{~h}$ in $<1 \% \mathrm{O}_{2}$ with no glucose, where pre-incubation with AICAR induced almost a 2fold increase in basal OCR and oxidative phosphorylation (the difference between OCR before and after addition of oligomycin) versus control, which were completely abolished by cll inhibition, as they were reduced to control values (Figure $5 d$, upper panel). In addition, AICAR significantly enhanced ECAR (Figure 5d, lower panel). Thus, cll harbors reserve activity that can be activated by AMPK, which allows the mitochondria to rapidly increase its energy output after energy deprivation.

To validate the results of the 3NP inhibitor on cll and RRC, we knocked down one of cll's assembly factors, succinate dehydrogenase assembly factor 1 (Sdhaf1). Figure 5e shows that knockdown of Sdhaf1 with a short hairpin (sh) RNA construct (Sdhaf1-sh) completely abrogated the RRC, with no significant effect on basal OCR at the lowest dose used of the adenoviral delivery vector (multiplicity of infection, moi 5) (Figure 5e). Increasing doses of Sdhaf1-sh, however, resulted in significant reduction $(20-35 \%)$ of basal OCR that was associated with almost an equivalent degree of cell death (Figure 5f). Since unassembled Sdha is a known source of excessive ROS formation, it explains the cell death observed at the higher doses of Sdhaf1-sh. The results confirm that holo-cll is indeed required for formation of RRC.

RRC and cll contribute to an increase in cell survival after energy deprivation. The RRC has been shown to correlate with cell survival in multiple cell types. ${ }^{1,2}$ Our goal here was to determine whether the different regulators of RRC that we have identified in myocytes had an effect on cell survival and whether cll had any role in the process. To address this, we incubated the cells with palmitate-BSA only, or glucose plus palmitate in the absence or presence of etomoxir, AICAR, 3NP or 3NP plus AICAR, before subjecting them to $<1 \% \mathrm{O}_{2}$ conditions in the absence of glucose for $6 \mathrm{~h}$ (lethal conditions). Significantly, in the presence of atmospheric $\mathrm{O}_{2}$ none of these treatments had an effect on cell survival or ROS production (Figures $6 \mathrm{a}-\mathrm{f}$, first and third panels from the left). After energy deprivation, followed by restoration for 30 min, cell death was $>90 \%$, accompanied by ROS production, when either glucose or fatty acid oxidation was inhibited glucose deprivation or etomoxir treatment, respectively (Figures $6 \mathrm{a}$ and $\mathrm{c}$, second and fourth panels from the left, and Figure $6 \mathrm{~g}$ ), versus $\sim 10 \%$ in control cells with glucose +palmitate (Figures $6 \mathrm{~b}$ and $\mathrm{g}$ ). Conversely, cells treated with AICAR exhibited $<1 \%$ cell death (Figure $6 \mathrm{~d}$, second panel and fourth panels from the left, and Figure $6 \mathrm{~g}$ ), which was lost by treatment with the cll inhibitor 3NP (Figure 6f, second and 


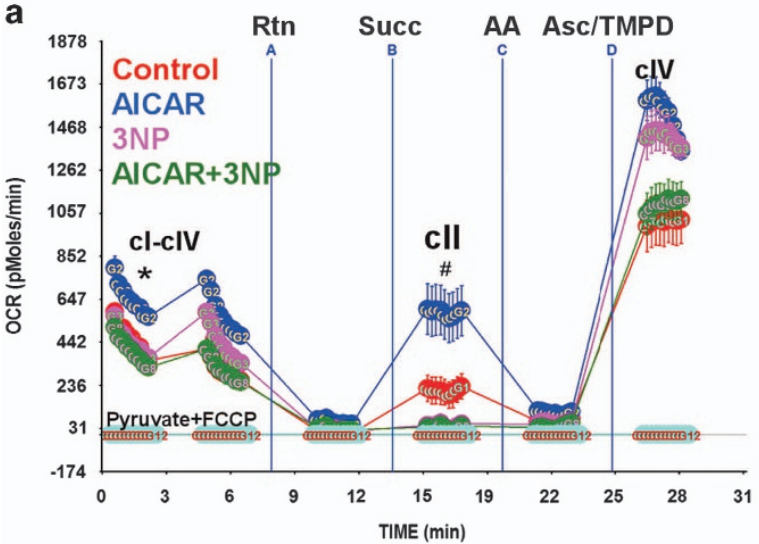

C
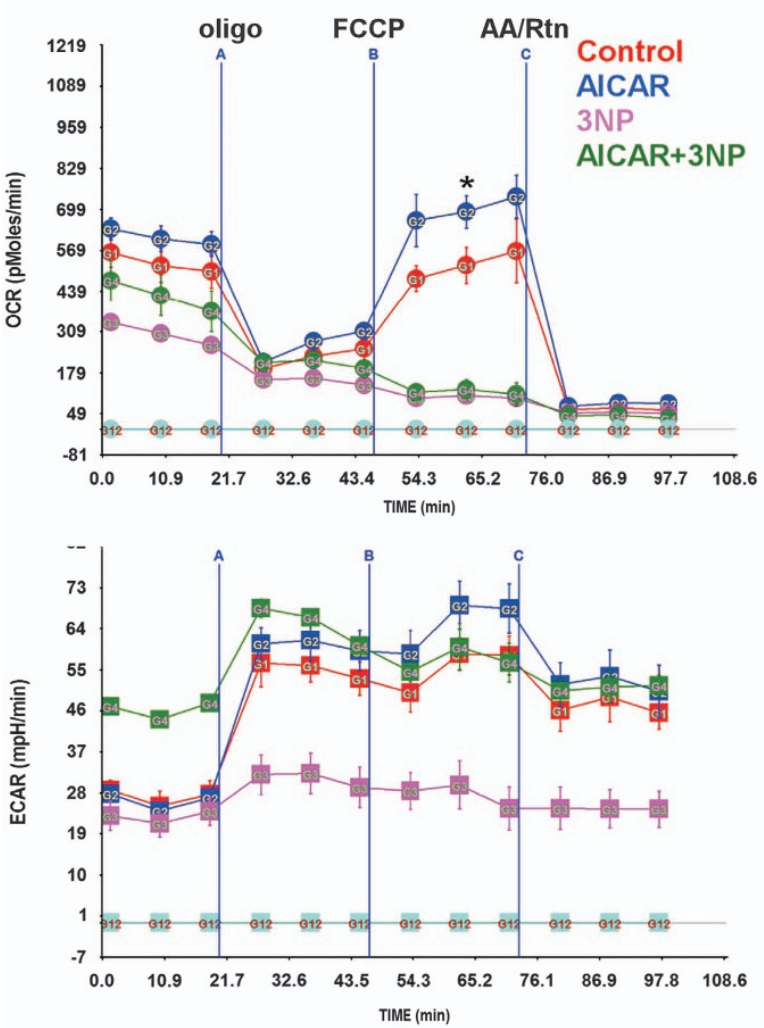

b

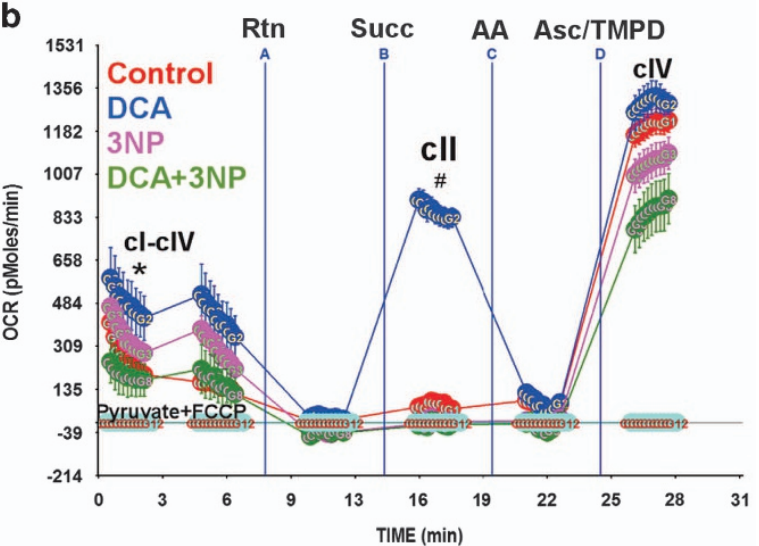

d
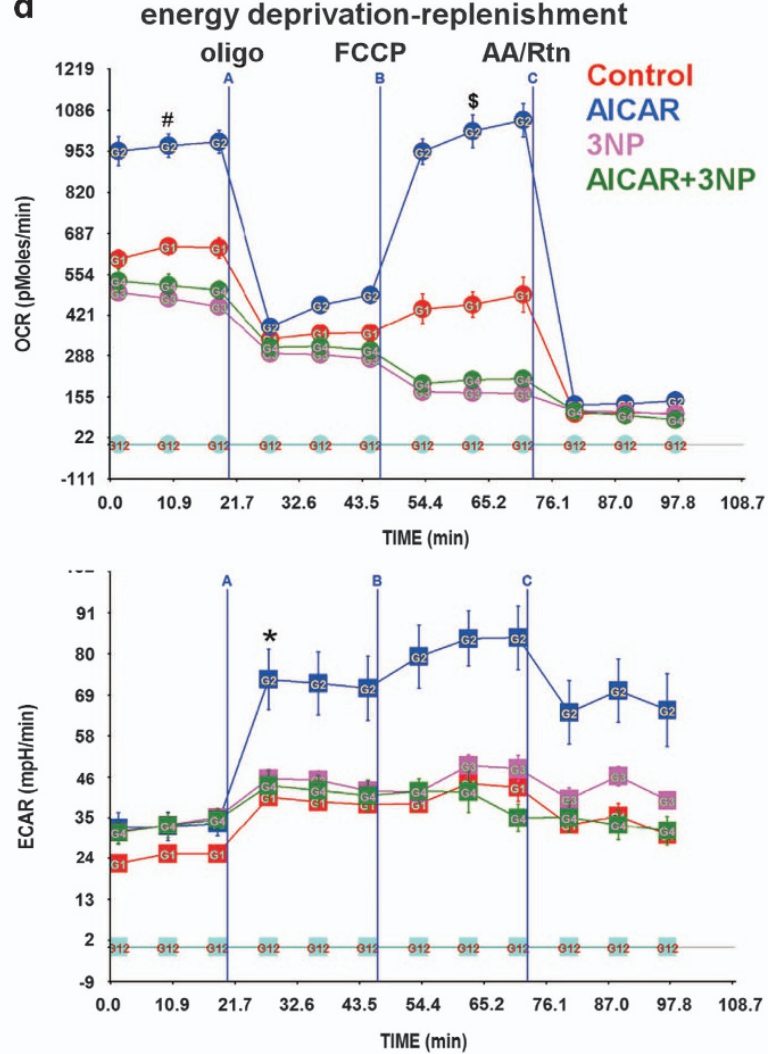

Figure 5 RRC is a product of cll activity and is utilized after energy deprivation. ( $\mathbf{a}$ and $\mathbf{b}$ ) Neonatal rat cardiac myocytes cultured in complete growth medium were treated with vehicle, $500 \mu \mathrm{M}$ AICAR (a) or $1 \mathrm{mM} \mathrm{DCA} \mathrm{(b),} \mathrm{for} 24 \mathrm{~h}$. Vehicle or $100 \mu \mathrm{M} 3 \mathrm{NP}$ was added for the last $1 \mathrm{~h}$ of this incubation. The cells were then permeabilized with $0.5 \mathrm{nM}$ PMP reagent ( $\mathrm{rPFO}$ ) in the presence of $10 \mathrm{mM}$ pyruvate, $2 \mathrm{mM}$ malate and $4 \mu \mathrm{M} \mathrm{FCCP}$, for $30 \mathrm{~min}$. The electron flow assay was then performed as described in Materials and Methods, as indicated, $n=4-6$, error bars represent S.E.M., each experiment was performed twice. Error bars represent S.E.M., ${ }^{*} P<0.05$ basal OCR for AICAR or DCA treated versus basal OCR for control, at the time point indicated; ${ }^{\#} P<0.05$ max OCR for AICAR or DCA treated versus max OCR for control, at the time point indicated. (c and $\mathbf{d}$ ) Neonatal rat cardiac myocytes cultured in complete growth medium were treated with vehicle or $500 \mu \mathrm{M}$ AICAR, for $24 \mathrm{~h}$. Vehicle or $100 \mu \mathrm{M} 3 \mathrm{NP}$ was added and the cells either remained in normoxic conditions (atmospheric $\left.\mathrm{O}_{2}\right)$ (c) or were exposed to hypoxia $\left(<1 \% \mathrm{O}_{2}\right)$ and glucose deprivation $(\mathbf{d})$, for $1 \mathrm{~h}$. At the end of this period, the medium was then replaced with serum-free XF medium containing $17.5 \mathrm{mM}$ glucose plus $100 \mu \mathrm{M}$ palmitate-BSA containing vehicle, $500 \mu \mathrm{M}$ AICAR, $100 \mu \mathrm{M} 3 \mathrm{NP}$ or $500 \mu \mathrm{M}$ AICAR plus $100 \mu \mathrm{M}$ $3 N P$, for $1 \mathrm{~h}$. The mitochondrial stress test was then performed as described in Materials and Methods, $n=4-6$, each experiment was performed twice. Error bars represent S.E.M., ${ }^{*} P<0.05$ max OCR versus basal OCR for AICAR-treated cells, at the time point indicated; ${ }^{\#} P<0.05$ basal OCR for AICAR treated versus basal OCR for control, at the time point indicated; ${ }^{\$} P<0.05$ max OCR for AICAR treated versus max OCR for control, at the time point indicated. (e) Neonatal rat cardiac myocytes (50 000-100 000/well) cultured in complete growth medium were infected with adenoviral vectors harboring a scrambled control sequence, shRNA targeting Sdhaf1 (5, 10, 15 moi) (Sdhaf1-sh), or an Sdhaf1 overexpressor (1,2 moi), for $24 \mathrm{~h}$. The medium was then replaced with serum-free XF medium containing $17.5 \mathrm{mM}$ glucose plus $100 \mu \mathrm{M}$ palmitate-BSA, for $1 \mathrm{~h}$. The mitochondrial stress test was then performed as described in Materials and Methods, $n=3-4$. Error bars represent S.E.M., ${ }^{*} P<0.05$ max OCR control versus max OCR for Sdhaf1-sh treated (all doses), at the time point indicated. (f) Cells treated as described in (e) were subjected to a live (green)/dead (red nuclei) assay and imaged 

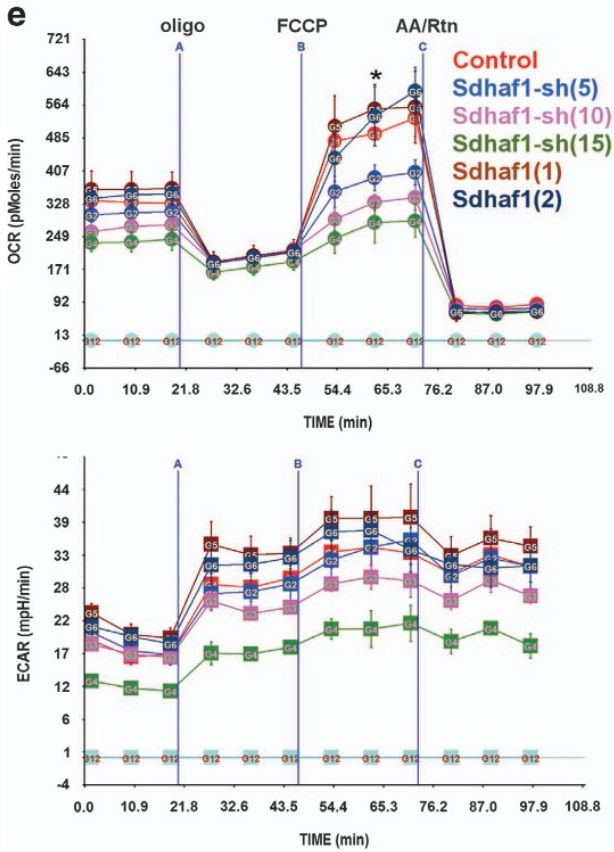

f

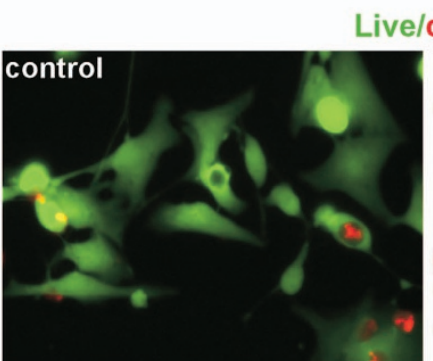

Live/dead
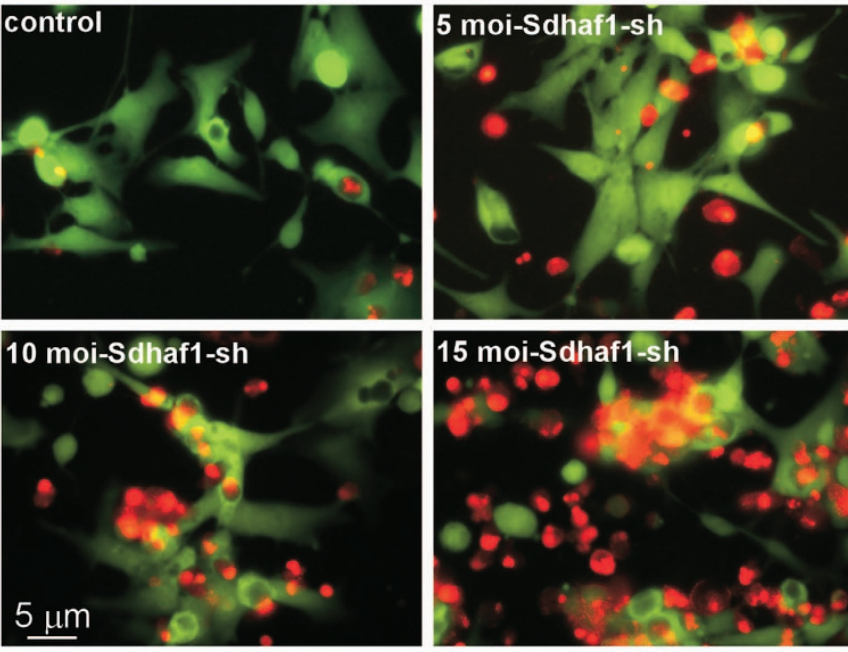

15 moi-Sdhaf 1 -sh

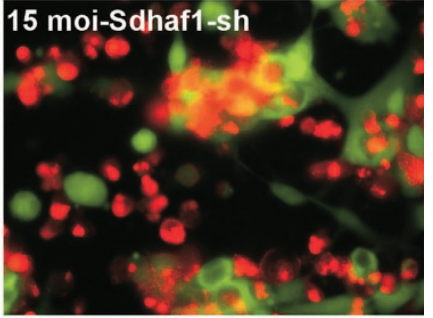

Figure 5 Continued

fourth panels from the left, and Figure $6 \mathrm{~g}$ ). Here, we used the membrane-impermeable propidium iodide staining for detection of nuclei in dead cells, ${ }^{21,22}$ which is dependent on a damaged plasma membrane for cell entry. This suggests that the mode of cell death detected in this experiment is mainly necrotic. We, thus, conclude that activation of cll and formation of an RRC allows the cells to minimize ROS formation and resist cell death due to energy deprivation, plausibly by increasing energy production after restoration of oxygen and nutrients, as seen in Figure 5.

Sirt3 is required for the development of RRC. Sirt3 is a mitochondrial sirtuin that regulates a plethora of proteins including NDUFA9 and complex I, ${ }^{23}$ as well as, complex II activity. ${ }^{24,25}$ We predicted that the effects of AMPK and PDH that regulate substrate flux into the TCA cycle and development of RRC might be mediated through the $\mathrm{NAD}^{+}$dependent Sirt3. To test this, we generated a short hairpin RNA targeting Sirt3 (Sirt3-sh). This construct, dose-dependently, abolished the RRC in cardiac myocytes, but had no effect on basal OCR (Figure 7a). Conversely, overexpressing Sirt3 resulted in a slight, but not significant increase in RRC, possibly due to saturating endogenous levels under these conditions. The results demonstrate that Sirt3 is required for the development of RRC, but has minimal effect on basal OCR. In agreement, Sirt3 knockdown also inhibited AICARand DCA-induced RRC (Figure 7b).

\section{Discussion}

A cell's energy production is directly linked to its demand. ${ }^{26,27}$ In this report, we show that cardiac myocytes operate on a fraction of their mitochondrial respiratory capacity under conditions where the preferred metabolic substrates are available. Although neonatal cardiac myocytes utilize glucose as the preferred substrate, a respiratory reserve will only develop in the presence of both glucose and fatty acids in this cell type, which suggests independent resources for basal OCR versus the RRC. We also show here, for the first time, that we can further increase the RRC by enhancing either glucose or fatty acid oxidation, via inhibiting pyruvate dehydrogenase kinases or stimulation of AMPK-Ppara axis, respectively. Conversely, hypoxia exhausts the RRC, which can be rescued by those measures. This suggested that the $\mathrm{RRC}$ is a product of a co-regulated increase in the TCA cycle and electron transport chain (ETC) activities.

Succinate dehydrogenase is the only enzyme in the TCA cycle that is also a component of the electron transport chain complex II, which suggested to us that it is well positioned to serve as a regulated source of the RRC. Indeed, our data show that an increase in glucose and/or fatty acid oxidation is sensed by cll, resulting in an increase in its activity. However, this spare cll activity that was recently observed ${ }^{28}$ remains unutilized under normal conditions, but becomes manifest when oxygen consumption is uncoupled from ATP production (Figures 1-4), or if there is an increase in energy demand (Figure 5). We show that inhibition of the Sdha subunit of cll by 3NP completely abrogates the RRC, proving that it is indeed a product of cll activity. These results were corroborated by a second independent approach that involved the knockdown of the assembly factor Sdhaf1, which, similarly, completely abolished RRC before it induced cell death. The cell death observed with Sdhaf1 knockdown is an expected effect of the excessive ROS produced by the disassembled Sdha subunit. ${ }^{16}$ Interestingly, in both approaches, the inhibition of cll resulted in only minimal inhibition of basal OCR (10-18\%), which is equivalent to the extent that cll contributes to the 

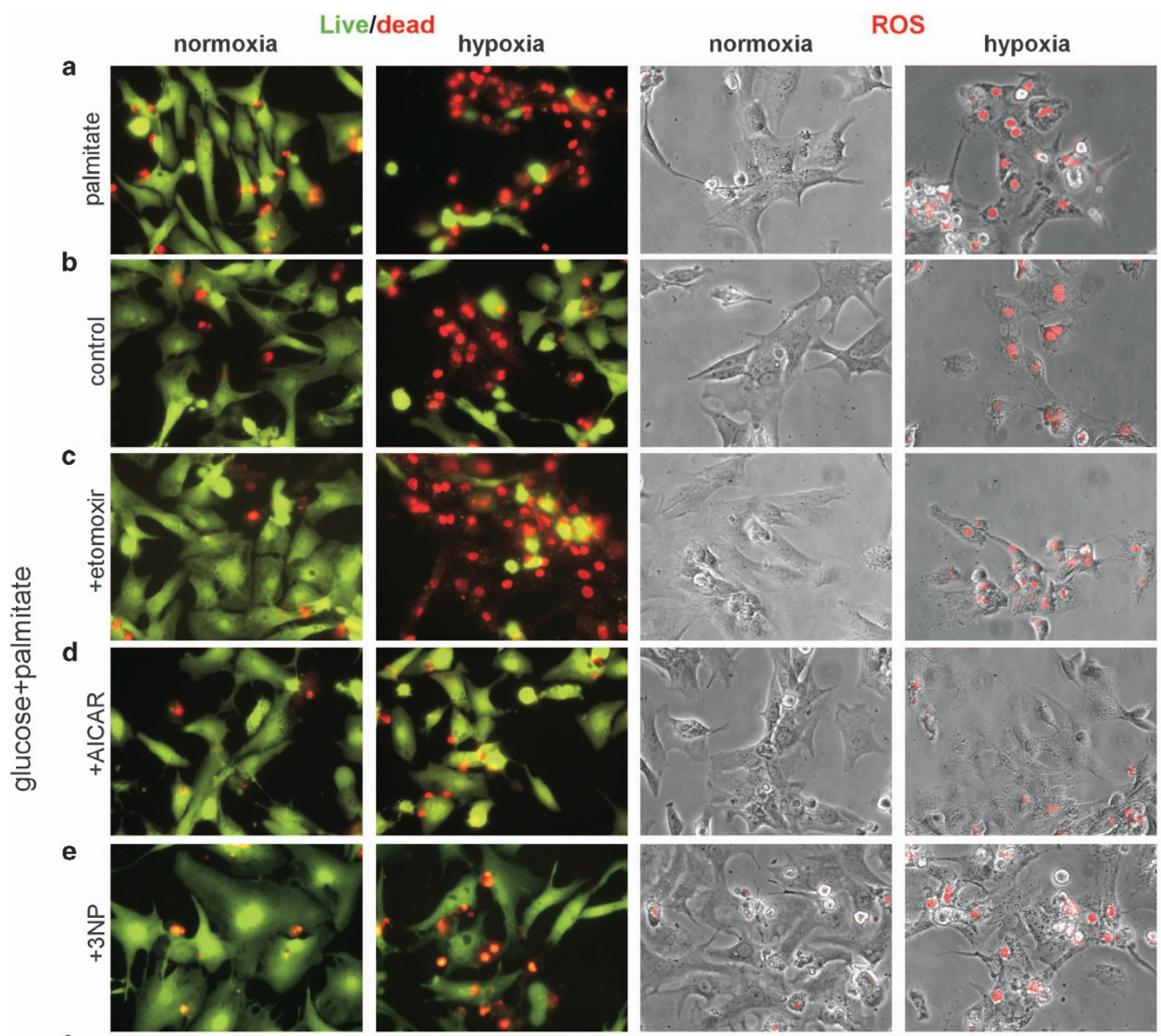

f
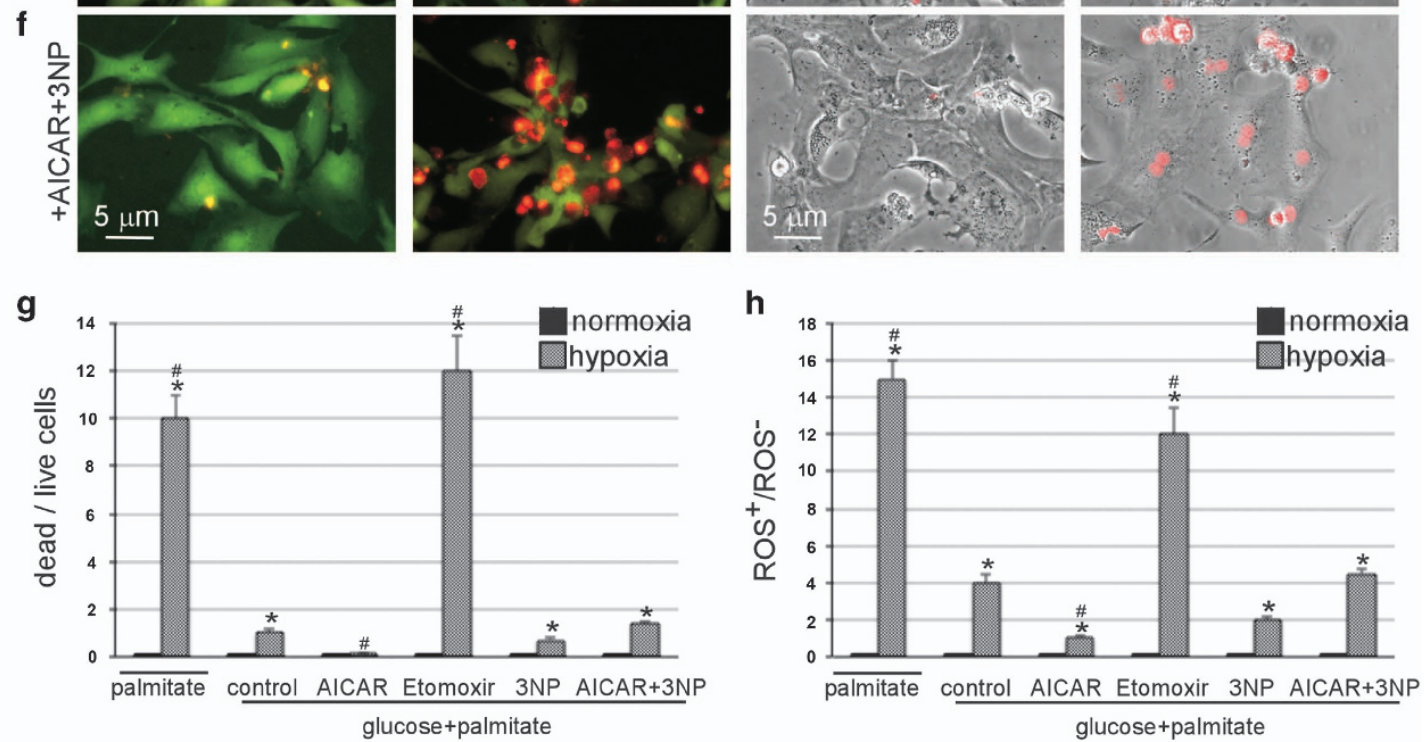

Figure 6 Conditions that are associated with a higher RRC are more resistant to cell death and produce less ROS. Cardiac myocytes were incubated in glucose+palmitate (b-f) except in (a) in palmitate only, (c)+etomoxir (Cpt1 inhibitor), (d) +AICAR (AMPK activator), for $24 \mathrm{~h}$ in normoxic conditions, (e) +3NP (cll inhibitor), for $1 \mathrm{~h}$ before hypoxia, (f) + AICAR+3NP (left and third from left panels a-f). Cells were then subjected to $6 \mathrm{~h}$ of $<0 \% \mathrm{O}_{2}$ for $6 \mathrm{~h}$ in the absence of glucose (second from the left and right panels a-f). This was followed by a live (green)/dead (red nuclei) assay (left and second from the left panels a-f) or ROS detection (red nuclei, right and third from the left panels a-f) that was visualized by live imaging of the cells. (g) The dead/live cell ratio was calculated and plotted $(n=3,100$ cells counted). Error bars represent S.E.M. and * $P<0.05$ versus normoxia. ${ }^{\#} P<0.05$ versus hypoxia (glucose+palmitate). (h) The ROS $/$ ROS $^{-}$cell ratio was calculated and plotted ( $n=2,60$ cells counted). Error bars represent S.E.M. and ${ }^{*} P<0.05$ versus normoxia. ${ }^{\#} P<0.05$ versus hypoxia (glucose+palmitate) 
a
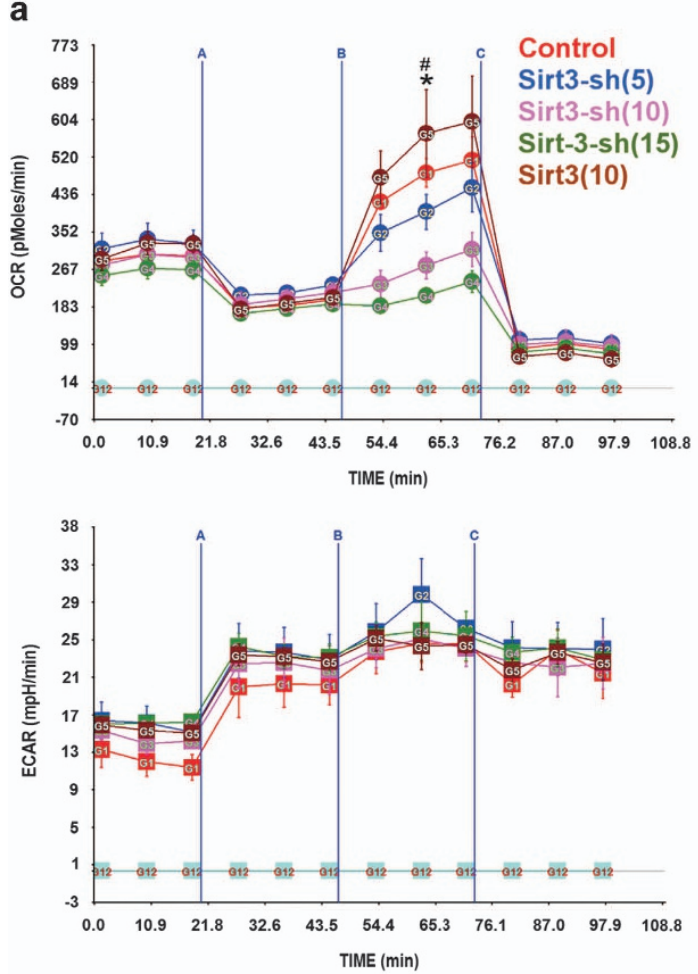

b
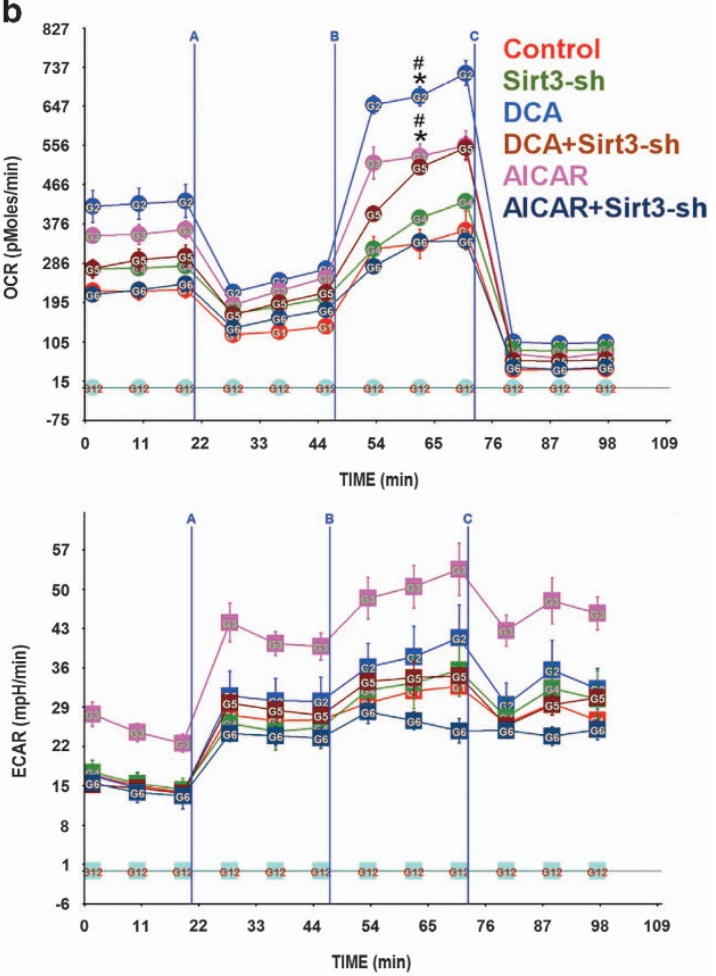

Figure 7 Sirt3 is required for development of RRC. (a) Neonatal rat cardiac myocytes cultured in complete growth medium were infected with adenoviral vectors harboring a scrambled control sequence, shRNA targeting Sirt3 (Sirt3-sh) (5, 10 or $15 \mathrm{moi}$ ) or a Sirt3 overexpressor (10 moi), for $24 \mathrm{~h}$. The medium was then replaced with serum-free XF medium containing $17.5 \mathrm{mM}$ glucose plus $100 \mu \mathrm{M}$ palmitate-BSA, for $1 \mathrm{~h}$. The mitochondrial stress test was then performed as described in Materials and Methods, $n=3-4$. Error bars represent S.E.M., ${ }^{*} P<0.05$ max OCR versus basal OCR for control, at the time point indicated; ${ }^{\sharp} P<0.05$ max OCR for control versus max OCR for Sirt3-sh treated (10 and 15 moi), at the time point indicated. (b and $\mathbf{c}$ ) Neonatal rat cardiac myocytes cultured in complete growth medium were treated with $500 \mu \mathrm{M} \mathrm{AICAR}$ (b) or $1 \mathrm{mM}$ DCA (c) in the presence of an adenoviral vector harboring a scrambled control sequence or a shRNA targeting Sirt3 (Sirt3-sh) (10 moi), for $24 \mathrm{~h}$. The medium was then replaced with serumfree XF medium containing $17.5 \mathrm{mM}$ glucose plus $100 \mu \mathrm{M}$ palmitate-BSA, $17.5 \mathrm{mM}$ glucose plus $100 \mu \mathrm{M}$ palmitate-BSA plus $1 \mathrm{mM}$ DCA or $17.5 \mathrm{mM}$ glucose plus $100 \mu \mathrm{M}$ palmitate-BSA plus $500 \mu \mathrm{M}$ AICAR, for $1 \mathrm{~h}$. The mitochondrial stress test was then performed as described in Materials and Methods, $n=3-4$. Error bars represent S.E.M., ${ }^{*} P<0.05$ max OCR versus basal OCR for DCA- or AICAR-treated cells, at the time point indicated; ${ }^{*} P<0.05$ max OCR for DCA or AICAR treated versus max OCR for DCA+Sirt3-sh or AICAR+Sirt3-sh treated, at the time point indicated

transfer of electrons from FADH and its oxidation via the ETC. Finally, we show that Sirt3 is required for formation of RRC, suggesting that it also regulates cll activity. This is indeed supported by the fact that Sdha has 13 lysine-acetylation sites that are deacetylated by Sirt3, and which regulate its activity. $^{24,25}$

Sdhaf1 gene has been recently discovered in association with infantile leukoencephalopathy, an infantile mitochondrial disease, where it harbors a missense point mutation, ${ }^{29}$ underscoring its critical regulatory function. This mutation is associated with a reduction in the cll holoenzyme (assembled complex) and an 70-80\% loss in Sdh and succinyl CoQ reductase activities in fibroblasts and muscle cells. Our data confirm that Sdhaf1 is critical for cll function in cardiac myocytes, as it is required for development of RRC, which enhances cell survival.

In summary, the work presents a novel mechanism by which cells can increase their energy output during an increase in demand, which includes assembly and activation of cll by the metabolic sensors and regulators, AMPK, PDH and Sirt3. In addition, we show that this parameter can be exploited to enhance the cell's tolerance to cell death signals.

\section{Materials and Methods}

Neonatal rat cardiac myocyte culture. Cardiac myocytes were prepared from 1- to 2-day-old Sprague-Dawley rats as previously described. ${ }^{30}$ The myocytes were treated in Dulbecco's modified essential medium/Ham F12 (1:1) supplemented with $10 \%$ fetal bovine serum (10\% FBS) and 1\% penicillin/ streptomycin or in Dulbecco's modified essential medium $(1 \mathrm{x})$ supplemented with glucose or palmitate-BSA, as indicated in the figure legends. Pharmacological treatments include etomoxir, dichloroacetate (DCA), Aicar and 3 nitropropionate (3NP) purchased from Sigma-Aldrich Corp. (St. Louis, MO, USA).

Human iPSC-cardiac myocyte culture. Human iCell cardiac myocytes (Cellular Dynamics International, Madison, WI, USA) were thawed and plated, as recommended by the manufacturer. After $48 \mathrm{~h}$, the medium was changed to Dulbecco's modified essential medium/Ham F12 (1:1) supplemented with $10 \%$ FBS and $1 \%$ penicillin/streptomycin. The cells were treated in this medium or in Dulbecco's modified essential medium $(1 x)$ supplemented with glucose or palmitate-BSA as indicated in the figure legends.

Adenovirus constructs. The human cDNA clones for Pdk1 (SC321678/ NM_002610), Pdk4 (SC118542/NM_002612), Sdhaf1 (NM_001042631) and Sirt3 (NM_012239) were purchased (Origene Technologies, Inc., Rockville, MD, USA) and cloned into recombinant adenovirus vectors, propagated, and titered as previously described by Graham and Prevec. ${ }^{31}$

Short hairpin constructs targeting succinate dehydrogenase assembly factor 1 (Sdhaf1) nt 31-50 of Rattus norvegicus NM_001177687.1 and Sirt3 nt 293-312 of Rattus norvegicus NM_001106313.2 were designed, synthesized (IDT), and cloned 
into adenoviral vectors. Cardiac myocytes were treated as indicated in the figure legends at an moi of 1-15.

Hypoxia. Neonatal rat cardiac myocytes or human iPSC-CM were subjected to hypoxia using a hypoxic chamber (Billups-Rothenberg, Inc., Del Mar, CA, USA) or (BioSpherix, Lacona, NY, USA). Either chamber is perfused with a gas mixture of $95 \% \mathrm{~N}_{2}$ and $5 \% \mathrm{CO}_{2}$ at $7 \mathrm{psi} / 12000 \mathrm{kPa}$ filling pressure, until a chamber oxygen concentration of $0.1 \%$ is reached. The chamber was then kept in a $37^{\circ} \mathrm{C}$ incubator.

Mitochondrial stress test. Neonatal cardiac myocytes (50 000-100 000 cells/well) or human iPS-derived cardiac myocytes $(60000 /$ well) were plated in 24-well Seahorse analyzer plates. OCR and ECAR were measured using the Seahorse XF24-3 Analyzer (Seahorse Bioscience, North Billerica, MA, USA), as recommended by the manufacturer. Briefly, after 20 (A), 45 (B), 70 (C) min of the start of measurements, the medium was injected with A, oligomycin; B, FCCP; C, antimycin A plus rotenone, as marked in the graphs. Three readings were taken after each injected compound and the results plotted as pMoles/min/well or $\mathrm{mpH} /$ $\mathrm{min} /$ well (y axis) versus time ( $\mathrm{x}$ axis).

Electron flow assay. OCR was measured in cardiac myocytes after cell permeabilization using XF PMP reagent (Seahorse Bioscience), as recommended by the manufacturer. Briefly, $10 \mathrm{mM}$ pyruvate plus $2 \mathrm{mM}$ malate are used as substrates, in addition to $4 \mu \mathrm{M}$ FCCP. Final concentrations of injected compounds were as follows: port $\mathrm{A}, 2 \mu \mathrm{M}$ rotenone; port $\mathrm{B}, 10 \mathrm{mM}$ succinate; port $\mathrm{C}, 4 \mu \mathrm{M}$ antimycin $\mathrm{A}$; port D, $10 \mathrm{mM}$ ascorbate plus $0.1 \mathrm{mM}$ TMPD.

Immunocytochemistry. Neonatal rat cardiac myocytes or human iPSC-CM were plated on gelatin-coated glass slides and treated, as indicated in the figure legends. The cells were then fixed, permeabilized, immunolabelled, mounted, and imaged by confocal microscopy. Anti-OxPhos Complex IV (Life Technologies, Grand Island, NY, USA) and anti-titin (Hybridoma Bank, University of lowa, lowa City, IA, USA) antibodies were used.

Quantitative PCR. Total RNA or DNA was extracted using TRIzol Reagent (Life Technologies). RNA was reversed transcribed to CDNA using High Capacity cDNA Reverse Transcription Kit (Applied Biosystems, a brand of Life Technologies). Quantitative PCR was performed using TaqMan gene expression assays (Applied Biosystems) on Applied Biosystems 7500 Real-Time PCR system for the following genes: 18S (Mm03928990_g1), Pdk1 (Rn00587598_m1), Pdk2 (Rn00446679_m1), Pdk3 (Rn01424337_m1), Pdk4 (Rn00585577_m1), Ppargc1 (Mm01208835_m1), PPARa (Rn00566193_m1), Cpt1 (Rn00682395_m1), tfam (Rn00580051_m1) and ND2 (Rn03296765_S1). Mitochondrial DNA was quantified using a primer/probe set for the mitochondrial D-loop (Applied Biosystems), as described by Nicklas et al..$^{32}$

Live/dead cell assay. Neonatal rat cardiac myocytes were plated on glass slides and treated, as indicated in the figure legends. Live/dead cells assay were performed using the LIVE/DEAD Viability/Cytotoxicity Kit (Life Technologies).

Superoxide assay. Neonatal rat cardiac myocytes were plated on glass slides and treated, as indicated in the figure legends. Superoxide production was measured using MitoSOX Red mitochondrial superoxide indicator (Life Technologies).

Statistical analysis. Significant differences between the mean of two sample groups were calculated using $T$-test (equal variance, two-tailed), where $P<0.05$ was considered as significant.

\section{Conflict of Interest}

The authors declare no conflict of interest.

Acknowledgements. We want to thank Dr. Junichi Sadoshima, Chairman of the Department of Cell Biology and Molecular Medicine, for his support. This work is partly supported by the National Institutes of Health (HL119726) to the corresponding authors.

\section{Author contributions}

JP has performed all the experiments in this manuscript, including all measurements of oxygen consumption rates and extracellular acidification rates using the Seahorse analyzer, live/dead assays, 2D gel electrophoresis, and qPCR for gene expression. In addition, JP has participated in the design of the experiments and in the preparation of the manuscript. MH cultured the primary cardiac myocytes used in all the experiments and constructed the adenoviral vectors used for gene delivery. MA designed, analyzed, interpreted, supervised all experiments, prepared all figures and wrote the manuscript.

1. Nickens KP, Wikstrom JD, Shirihai OS, Patierno SR, Ceryak S. A bioenergetic profile of nontransformed fibroblasts uncovers a link between death-resistance and enhanced spare respiratory capacity. Mitochondrion 2013; 13: 662-667.

2. Yadava N, Nicholls DG. Spare respiratory capacity rather than oxidative stress regulates glutamate excitotoxicity after partial respiratory inhibition of mitochondrial complex I with rotenone. J Neurosci 2007; 27: 7310-7317.

3. Choi SW, Gerencser AA, Nicholls DG. Bioenergetic analysis of isolated cerebrocortical nerve terminals on a microgram scale: spare respiratory capacity and stochastic mitochondrial failure. J Neurochem 2009; 109: 1179-1191.

4. Flynn JM, Choi SW, Day NU, Gerencser AA, Hubbard A, Melov S. Impaired spare respiratory capacity in cortical synaptosomes from Sod2 null mice. Free Radic Biol Med 2011; 50: 866-873.

5. Hill BG, Benavides GA, Lancaster Jr JR, Ballinger S, Dell'Italia L, Jianhua Z et al. Integration of cellular bioenergetics with mitochondrial quality control and autophagy. Biol Chem 2012; 393: $1485-1512$.

6. Nicholls DG. Spare respiratory capacity, oxidative stress and excitotoxicity. Biochem Soc Trans 2009; 37: 1385-1388.

7. Sansbury BE, Jones SP, Riggs DW, Darley-Usmar VM, Hill BG. Bioenergetic function in cardiovascular cells: the importance of the reserve capacity and its biological regulation. Chem Biol Interact 2011; 191: 288-295.

8. Siddiqui A, Rivera-Sanchez S, Castro Mdel R, Acevedo-Torres K, Rane A, Torres-Ramos CA et al. Mitochondrial DNA damage is associated with reduced mitochondrial bioenergetics in Huntington's disease. Free Radic Biol Med 2012; 53: 1478-1488.

9. van der Windt GJ, Everts B, Chang CH, Curtis JD, Freitas TC, Amiel E et al. Mitochondrial respiratory capacity is a critical regulator of $\mathrm{CD} 8+\mathrm{T}$ cell memory development. Immunity 2012; 36: 68-78.

10. Bourgeron T, Rustin P, Chretien D, Birch-Machin M, Bourgeois M, Viegas-Pequignot E et al. Mutation of a nuclear succinate dehydrogenase gene results in mitochondrial respiratory chain deficiency. Nat Genet 1995; 11: 144-149.

11. Jain-Ghai S, Cameron JM, Al Maawali A, Blaser S, MacKay N, Robinson B et al. Complex II deficiency-a case report and review of the literature. Am J Med Genet A 2013; 161A: 285-294.

12. Walker DW, Hajek P, Muffat J, Knoepfle D, Cornelison S, Attardi G et al. Hypersensitivity to oxygen and shortened lifespan in a Drosophila mitochondrial complex II mutant. Proc Natl Acad Sci USA 2006; 103: 16382-16387.

13. Wojtovich AP, Brookes PS. The complex II inhibitor atpenin A5 protects against cardiac ischemia-reperfusion injury via activation of mitochondrial KATP channels. Basic Res Cardiol 2009; 104: 121-129.

14. Albayrak T, Grimm S. A high-throughput screen for single gene activities: isolation of apoptosis inducers. Biochem Biophys Res Commun 2003; 304: 772-776.

15. Grimm S. Respiratory chain complex II as general sensor for apoptosis. Biochim Biophys Acta 2013; 1827: 565-572.

16. Lemarie A, Huc L, Pazarentzos E, Mahul-Mellier AL, Grimm S. Specific disintegration of complex II succinate:ubiquinone oxidoreductase links $\mathrm{pH}$ changes to oxidative stress for apoptosis induction. Cell Death Differ 2011; 18: 338-349.

17. Quinlan CL, Orr AL, Perevoshchikova, Treberg JR, Ackrell BA, Brand MD. Mitochondrial complex II can generate reactive oxygen species at high rates in both the forward and reverse reactions. J Biol Chem 2012; 287: 27255-27264.

18. Whitehouse S, Cooper RH, Randle PJ. Mechanism of activation of pyruvate dehydrogenase by dichloroacetate and other halogenated carboxylic acids. Biochem J 1974; 141: 761-774.

19. Zaha VG, Young LH. AMP-activated protein kinase regulation and biological actions in the heart. Circ Res 2012; 111: 800-814.

20. Dobrzyn P, Dobrzyn A, Miyazaki M, Cohen P, Asilmaz E, Hardie DG et al. Stearoyl-CoA desaturase 1 deficiency increases fatty acid oxidation by activating AMP-activated protein kinase in liver. Proc Natl Acad Sci USA 2004; 101: 6409-6414.

21. Regula KM, Baetz D, Kirshenbaum LA. Nuclear factor-kappaB represses hypoxia-induced mitochondrial defects and cell death of ventricular myocytes. Circulation 2004; 110: 3795-3802.

22. Regula KM, Ens K, Kirshenbaum LA. Inducible expression of BNIP3 provokes mitochondrial defects and hypoxia-mediated cell death of ventricular myocytes. Circ Res 2002; 91: 226-231.

23. Abdellatif M. Sirtuins and pyridine nucleotides. Circ Res 2012; 111: 642-656.

24. Cimen H, Han MJ, Yang Y, Tong Q, Koc H, Koc EC. Regulation of succinate dehydrogenase activity by SIRT3 in mammalian mitochondria. Biochemistry 2010; 49: 304-311.

25. Finley LW, Haas W, Desquiret-Dumas V, Wallace DC, Procaccio V, Gygi SP et al. Succinate dehydrogenase is a direct target of sirtuin 3 deacetylase activity. PLoS One 2011; 6: e23295. 
26. van Beek $\mathrm{JH}$. Adenine nucleotide-creatine-phosphate module in myocardial metabolic system explains fast phase of dynamic regulation of oxidative phosphorylation. Am J Physiol Cell Physiol 2007; 293: C815-C829.

27. Vendelin M, Lemba M, Saks VA. Analysis of functional coupling: mitochondrial creatine kinase and adenine nucleotide translocase. Biophys $J$ 2004; 87: 696-713.

28. Wust RC, Helmes M, Stienen GJ. Rapid changes in NADH and flavin autofluorescence in rat cardiac trabeculae reveal large mitochondrial complex II reserve capacity. J Physiol 2015; 593: $1829-1840$

29. Ghezzi D, Goffrini P, Uziel G, Horvath R, Klopstock T, Lochmuller H et al. SDHAF1, encoding a LYR complex-II specific assembly factor, is mutated in SDH-defective infantile leukoencephalopathy. Nat Genet 2009; 41: 654-656.

30. Abdellatif M, Mcllelan WR, Schneider MD. p21 Ras as a governer of global gene expression. J Biol Chem 1994; 269: 15423-15426.

31. Graham FL, Prevec L. Methods in Molecular Biology, vol. 7. The Humana Press Inc.: Clifton, NJ, 1991.
32. Nicklas JA, Brooks EM, Hunter TC, Single R, Branda RF. Development of a quantitative PCR (TaqMan) assay for relative mitochondrial DNA copy number and the common mitochondrial DNA deletion in the rat. Environ Mol Mutagen 2004; 44: 313-320.

(c) (i)

Cell Death and Disease is an open-access journal published by Nature Publishing Group. This work is licensed under a Creative Commons Attribution 4.0 International License. The images or other third party material in this article are included in the article's Creative Commons license, unless indicated otherwise in the credit line; if the material is not included under the Creative Commons license, users will need to obtain permission from the license holder to reproduce the material. To view a copy of this license, visit http://creativecommons.org/licenses/by/4.0/

Supplementary Information accompanies this paper on Cell Death and Disease website (http://www.nature.com/cddis) 